Submitted to the Annals of Statistics

Supplement to

\title{
JOINT ASYMPTOTICS FOR SEMI-NONPARAMETRIC REGRESSION MODELS WITH PARTIALLY LINEAR STRUCTURE
}

\author{
By Guang Cheng $\ddagger$ And Zuofeng Shang ${ }^{\S}$ \\ Department of Statistics, Purdue University \\ MARCH 7, 2015
}

In this document, we give the proofs of several results that were not given in the main text of this paper. The reference labels of the equations, Theorems, Propositions and Lemmas in this document are consistent with those in the main text of the paper.

This supplement document is organized as follows. Section S.1 proves that $\|(\theta, g)\|$ defies a valid norm of the semiparametric function space. Section S.2 proves the existence of the operator $W_{\lambda}$. Sections S.3 and S.4 contain the proofs of Propositions 2.1 and 2.2, respectively. Section S.5 includes the proof of Lemma 2.4. Section S.6 contains the statement and proof of a useful concentration inequality, i.e., Lemma S.3. In Section S.7, we prove Proposition 2.5 , i.e., the rates of convergence of the estimate $\widehat{f}_{n, \lambda}$. In Section S.8, we prove the joint Bahadur representation, i.e., Theorem 2.6. Sections S.9 and S.10 contain the proofs of Corollary 3.2 and Proposition 4.2, respectively. In Section S.11, we prove the restricted joint Bahadur representation, i.e., Theorem 4.3. Section S.12 contains the proof of Corollary 4.5. Section S.13 contains the proof of Corollary 4.7. In Section S.14, we verify the equivalence between the asymptotic variance of our parametric estimator and that in [23]. In Section S.15, we present reasonable situations where Example 5.3 satisfies assumptions in Theorems 3.1 and 4.4. Section S.16 presents an algorithm in computing the constrained estimate under a local hypothesis.

\section{S.1. Validity of Norm.}

Lemma S.1. Under Assumption A3, $\|(\theta, g)\|$ defines a valid norm on the semiparametric space $\mathbb{R}^{p} \times H^{m}(0,1)$.

\footnotetext{
${ }^{\ddagger}$ Corresponding Author. Associate Professor, Research Sponsored by NSF, DMS0906497, CAREER Award DMS-1151692, Simons Foundation 35266

${ }^{\S}$ Visiting Assistant Professor
} 
Proof of Lemma S.1. The absolute scalability and subadditivity of $\|(\theta, g)\|$ is obvious. Next we assume $\|(\theta, g)\|=0$, for $\theta \in \mathbb{R}^{p}$ and $g \in H^{m}(0,1)$, and show $(\theta, g)=0$. Let $\Omega$ be defined as in Assumption A3 of the manuscript. We have

$$
\begin{aligned}
0= & \|(\theta, g)\|^{2} \\
= & E\left\{I(U)\left(X^{T} \theta+g(Z)\right)^{2}\right\}+\lambda \int_{0}^{1}\left|g^{(m)}(z)\right|^{2} d z \\
= & E\left\{I(U)\left((X-G(Z))^{T} \theta+G(Z)^{T} \theta+g(Z)\right)^{2}\right\}+\lambda \int_{0}^{1}\left|g^{(m)}(z)\right|^{2} d z \\
= & \theta^{T} E\left\{I(U)(X-G(Z))(X-G(Z))^{T}\right\} \theta+E\left\{I(U)\left(G(Z)^{T} \theta+g(Z)\right)^{2}\right\} \\
& +\lambda \int_{0}^{1}\left|g^{(m)}(z)\right|^{2} d z \\
\geq & \theta^{T} \Omega \theta .
\end{aligned}
$$

Since $\Omega$ is positive definite, $\theta=0$. Thus, from the above derivations, $E\left\{I(U) g(Z)^{2}\right\}=$ $E\left\{B(Z) g(Z)^{2}\right\}=\int_{0}^{1} B(z) \pi(z) g(z)^{2} d z=0$. Since $\pi(z)$ and $B(z)$ are positivevalued for all $z \in[0,1]$ (Assumption A1 assumes that $B(z)$ is positive for all $z \in[0,1])$, we have $g=0$. Thus, the conclusion of Lemma S.1 holds.

\section{S.2. Existence of $W_{\lambda}$.}

Lemma S.2. There exists a bounded linear operator $W_{\lambda}$ from $H^{m}(0,1)$ to $H^{m}(0,1)$ such that for any $g, g_{1} \in H^{m}(0,1)$,

$$
\left\langle W_{\lambda} g, g_{1}\right\rangle_{1}=\lambda J\left(g, g_{1}\right) .
$$

Proof of Lemma S.2. Note that, endowed with $\|\cdot\|_{1}, H^{m}(0,1)$ is a RKHS. Consider the bilinear form $L\left(g, g_{1}\right) \equiv \lambda J\left(g, g_{1}\right)$, defined upon $\left(g, g_{1}\right) \in$ $H^{m}(0,1) \times H^{m}(0,1)$.

For any $g \in H^{m}(0,1)$, define a functional $L_{g}$ by

$$
L_{g}\left(g_{1}\right)=L\left(g, g_{1}\right) \text {, for any } g_{1} \in H^{m}(0,1) .
$$

Therefore, $L_{g}: H^{m}(0,1) \mapsto \mathbb{R}$ is a linear functional. The boundedness of $L_{g}$ follows by the following trivial fact: for any $g_{1} \in H^{m}(0,1)$, by CauchySchwartz inequality,

$\left|L_{g}\left(g_{1}\right)\right|=\left|\lambda J\left(g, g_{1}\right)\right| \leq \lambda^{1 / 2} J^{1 / 2}(g, g) \cdot\left(\lambda J\left(g_{1}, g_{1}\right)\right)^{1 / 2} \leq \lambda^{1 / 2} J^{1 / 2}(g, g)\left\|g_{1}\right\|_{1}$.

In summary, $L_{g}$ is a bounded linear functional on the Hilbert space $H^{m}(0,1)$. By Riesz representation theorem, there exists an unique element $\tilde{g} \in H^{m}(0,1)$ 
such that for any $g_{1} \in H^{m}(0,1)$,

$$
L_{g}\left(g_{1}\right)=\left\langle\tilde{g}, g_{1}\right\rangle_{1}
$$

Define $W_{\lambda} g=\tilde{g}$. Thus, $W_{\lambda}$ actually defines a map on $H^{m}(0,1)$ by mapping any $g \in H^{m}(0,1)$ to the element $\tilde{g}$ that satisfies (S.1). Hence, $\left\langle W_{\lambda} g, g_{1}\right\rangle_{1}=$ $L_{g}\left(g_{1}\right)=\lambda J\left(g, g_{1}\right)$ for any $g_{1} \in H^{m}(0,1)$.

The linearity of $W_{\lambda}$ follows by the linearity of the bilinear form $L$. Specifically, for any $g, g^{\prime} \in H^{m}(0,1)$ and any $a, b \in \mathbb{R}, W_{\lambda}\left(a g+b g^{\prime}\right)$ is an element in $H^{m}(0,1)$ satisfying that

$$
L_{a g+b g^{\prime}}\left(g_{1}\right)=\left\langle W_{\lambda}\left(a g+b g^{\prime}\right), g_{1}\right\rangle_{1}, \text { for any } g_{1} \in H^{m}(0,1) .
$$

On the other hand, for any $g_{1} \in H^{m}(0,1)$,

$$
\begin{aligned}
L_{a g+b g^{\prime}}\left(g_{1}\right) & =\left\langle a g+b g^{\prime}, g_{1}\right\rangle_{1} \\
& =a\left\langle g, g_{1}\right\rangle_{1}+b\left\langle g^{\prime}, g_{1}\right\rangle_{1} \\
& =a L_{g}\left(g_{1}\right)+b L_{g^{\prime}}\left(g_{1}\right)=\left\langle a W_{\lambda} g+b W_{\lambda} g^{\prime}, g_{1}\right\rangle_{1} .
\end{aligned}
$$

Thus, we have $\left\langle W_{\lambda}\left(a g+b g^{\prime}\right), g_{1}\right\rangle_{1}=\left\langle a W_{\lambda} g+b W_{\lambda} g^{\prime}, g_{1}\right\rangle_{1}$, for any $g_{1} \in$ $H^{m}(0,1)$. This implies $W_{\lambda}\left(a g+b g^{\prime}\right)=a W_{\lambda} g+b W_{\lambda} g^{\prime}$, i.e., the linearity of $W_{\lambda}$.

We next show the boundedness of $W_{\lambda}$ by the following derivations: for any $g \in H^{m}(0,1)$, by Cauchy-Schwartz inequality,

$$
\begin{aligned}
\left\|W_{\lambda} g\right\|_{1} & =\sup _{\substack{g_{1} \in H^{m}(0,1) \\
\left\|g_{1}\right\|_{1}=1}}\left|\left\langle W_{\lambda} g, g_{1}\right\rangle_{1}\right| \\
& =\sup _{\substack{g_{1} \in H^{m}(0,1) \\
\left\|g_{1}\right\|_{1}=1}}\left|\lambda J\left(g, g_{1}\right)\right| \\
& \leq \lambda^{1 / 2} J^{1 / 2}(g, g) \sup _{\substack{g_{1} \in H^{m}(0,1) \\
\left\|g_{1}\right\|_{1}=1}} \sqrt{\lambda J\left(g_{1}, g_{1}\right)} \\
& \leq \lambda^{1 / 2} J^{1 / 2}(g, g) \underset{\substack{\sup _{1} \in H^{m}(0,1) \\
\left\|g_{1}\right\|_{1}=1}}{\left\|g_{1}\right\|_{1}} \\
& =\lambda^{1 / 2} J^{1 / 2}(g, g) \leq\|g\|_{1} .
\end{aligned}
$$

Therefore, $W_{\lambda}$ is bounded with the operator norm no larger than one.

S.3. Proof of Proposition 2.1. The idea is to use property (2.6) in the main paper to solve $H_{u}$ and $T_{u}$. We use $E\{\}$ to denote the expectation with 
respect to the random pair $U=(X, Z)$. For any $f=(\theta, g) \in \mathcal{H}$, by $(2.5)$ in the main paper

$$
\begin{aligned}
& \left\langle\left(H_{u}, T_{u}\right), f\right\rangle \\
= & E\left\{I(U)\left(X^{T} \theta+g(Z)\right)\left(X^{T} H_{u}+T_{u}(Z)\right)\right\}+\lambda J\left(T_{u}, g\right) \\
= & E\left\{I(U)\left(\theta^{T} X X^{T} H_{u}+\theta^{T} X T_{u}(Z)+g(Z) X^{T} H_{u}+g(Z) T_{u}(Z)\right)\right\} \\
& +\lambda J\left(T_{u}, g\right) \\
= & \theta^{T} E\left\{I(U) X X^{T}\right\} H_{u}+\theta^{T} E\left\{I(U) X T_{u}(Z)\right\}+E\left\{I(U) g(Z) X^{T}\right\} H_{u} \\
& +E\left\{I(U) g(Z) T_{u}(Z)\right\}+\lambda J\left(T_{u}, g\right) \\
= & \theta^{T}\left(E\left\{I(U) X X^{T}\right\} H_{u}+E_{Z}\left\{B(Z) G(Z) T_{u}(Z)\right\}\right)+E_{Z}\left\{B(Z) G(Z)^{T} g(Z)\right\} H_{u} \\
& +\left\langle g, T_{u}\right\rangle_{1} \\
= & \left.\theta^{T}\left(E\left\{I(U) X X^{T}\right\} H_{u}+E_{Z}\left\{B(Z) G(Z) T_{u}(Z)\right\}\right\}\right)+\left\langle A^{T} H_{u}, g\right\rangle_{1}+\left\langle g, T_{u}\right\rangle_{1} \\
= & \theta^{T}\left(E\left\{I(U) X X^{T}\right\} H_{u}+E_{Z}\left\{B(Z) G(Z) T_{u}(Z)\right\}\right)+\left\langle A^{T} H_{u}+T_{u}, g\right\rangle_{1} \\
= & \theta^{T} x+\left\langle K_{z}, g\right\rangle_{1} .
\end{aligned}
$$

Therefore, by arbitrariness of $\theta$ and $g$, we have the equations

$$
\left\{\begin{array}{c}
E\left\{I(U) X X^{T}\right\} H_{u}+E_{Z}\left\{B(Z) G(Z) T_{u}(Z)\right\}=x \\
A^{T} H_{u}+T_{u}=K_{z}
\end{array}\right.
$$

Note that from the second equation we have $T_{u}=K_{z}-A^{T} H_{u}$. Then substitute it to the first equation, we have

$$
\begin{aligned}
& x \\
= & E\left\{I(U) X X^{T}\right\} H_{u}+E\left\{B(Z) G(Z)\left(K_{z}(Z)-A(Z)^{T} H_{u}\right)\right\} \\
= & E\left\{I(U) X X^{T}\right\} H_{u}+E\left\{B(Z) G(Z) K_{z}(Z)\right\} \\
& -E\left\{B(Z) G(Z) G(Z)^{T}\right\} H_{u}+E\left\{B(Z) G(Z)(G(Z)-A(Z))^{T}\right\} H_{u} \\
= & \left(\Omega+\Sigma_{\lambda}\right) H_{u}+E\left\{B(Z) G(Z) K_{z}(Z)\right\} \\
= & \left(\Omega+\Sigma_{\lambda}\right) H_{u}+A(z),
\end{aligned}
$$

where recall that $\Sigma_{\lambda}=E\left\{B(Z) G(Z)(G(Z)-A(Z))^{T}\right\}$ is a $p \times p$ matrix. In view of Lemma A.1 in the main paper, $\Sigma_{\lambda}$ is asymptotically negligible, thus, $\Omega+\Sigma_{\lambda}$ is asymptotically positive definite. This gives us

$$
H_{u}=\left(\Omega+\Sigma_{\lambda}\right)^{-1}(x-A(z)),
$$

Then from the second equation we have

$$
T_{u}=K_{z}-A^{T} H_{u}=K_{z}-A^{T}\left(\Omega+\Sigma_{\lambda}\right)^{-1}(x-A(z)) .
$$

By this way we have constructed explicitly the $R_{u}=\left(H_{u}, T_{u}\right)$ such that for any $u=(x, z) \in \mathcal{U}$ and any $f=(\theta, g) \in \mathcal{H}$,

$$
\left\langle R_{u}, f\right\rangle=x^{T} \theta+g(z) .
$$


S.4. Proof of Proposition 2.2. The idea is similar to the proof of (2.6) in the main paper. Denote $P_{\lambda} f=\left(H_{g}^{*}, T_{g}^{*}\right)$. Then we will use property $(2.7)$ in the main paper to solve $H_{g}^{*}$ and $T_{g}^{*}$. From (2.7) in the main paper,

$$
\begin{aligned}
& \left\langle P_{\lambda} f, \widetilde{f}\right\rangle \\
= & E\left\{I(U)\left(X^{T} H_{g}^{*}+T_{g}^{*}(Z)\right)\left(X^{T} \widetilde{\theta}+\widetilde{g}(Z)\right)\right\}+\lambda J\left(T_{g}^{*}, \widetilde{g}\right) \\
= & \left(H_{g}^{*}\right)^{T} E\left\{I(U) X X^{T}\right\} \widetilde{\theta}+\left(H_{g}^{*}\right)^{T} E\{I(U) X \widetilde{g}(Z)\}+E\left\{I(U) T_{g}^{*}(Z) X^{T}\right\} \widetilde{\theta} \\
& +E\left\{I(U) T_{g}^{*}(Z) \widetilde{g}(Z)\right\}+\lambda J\left(T_{g}^{*}, \widetilde{g}\right) \\
= & \left(\left(H_{g}^{*}\right)^{T} E\left\{I(U) X X^{T}\right\}+E\left\{B(Z) G(Z)^{T} T_{g}^{*}(Z)\right\}\right) \widetilde{\theta} \\
& +\left(H_{g}^{*}\right)^{T} E\{B(Z) G(Z) \widetilde{g}(Z)\}+\left\langle T_{g}^{*}, \widetilde{g}\right\rangle_{1} \\
= & \left(\left(H_{g}^{*}\right)^{T} E\left\{I(U) X X^{T}\right\}+E\left\{B(Z) G(Z)^{T} T_{g}^{*}(Z)\right\}\right) \widetilde{\theta}+\left\langle\left(H_{g}^{*}\right)^{T} A, \widetilde{g}\right\rangle_{1}+\left\langle T_{g}^{*}, \widetilde{g}\right\rangle_{1} \\
= & \left\langle W_{\lambda} g, \widetilde{g}\right\rangle_{1} .
\end{aligned}
$$

So we get the equations

$$
\left\{\begin{array}{c}
\left(H_{g}^{*}\right)^{T} E\left\{I(U) X X^{T}\right\}+E\left\{B(Z) G(Z)^{T} T_{g}^{*}(Z)\right\}=0 \\
\left(H_{g}^{*}\right)^{T} A+T_{g}^{*}=W_{\lambda} g .
\end{array}\right.
$$

The solution of the above equations is

$$
\left\{\begin{array}{c}
H_{g}^{*}=-\left(\Omega+\Sigma_{\lambda}\right)^{-1} E\left\{B(Z) G(Z)\left(W_{\lambda} g\right)(Z)\right\} \\
T_{g}^{*}=E\left\{B(Z) G(Z)^{T}\left(W_{\lambda} g\right)(Z)\right\}\left(\Omega+\Sigma_{\lambda}\right)^{-1} A+W_{\lambda} g .
\end{array}\right.
$$

So $P_{\lambda} f=\left(H_{g}^{*}, T_{g}^{*}\right)$ satisfies property $(2.7)$ in the main paper. Obviously $P_{\lambda}$ is linear and self-adjoint, i.e., $\left\langle P_{\lambda} f, \widetilde{f}\right\rangle=\left\langle f, P_{\lambda} \widetilde{f}\right\rangle$. The boundedness of $P_{\lambda}$ follows from the inequality (2.14) in the main paper.

S.5. Proof of Lemma 2.4. For any $u=(x, z) \in \mathcal{U},\left|\left\langle R_{u}, f\right\rangle\right| \leq\left\|R_{u}\right\| \cdot\|f\|$, so we only need to find the bound for $\left\|R_{u}\right\|$. By definition of $R_{u}$,

$$
\begin{aligned}
& \left\langle R_{u}, R_{u}\right\rangle \\
= & x^{T} x_{u}+T_{u}(z) \\
= & K(z, z) \\
& +\left(x-E\left\{B(Z) G(Z) K_{z}(Z)\right\}\right)^{T}\left(\Omega+\Sigma_{\lambda}\right)^{-1}\left(x-E\left\{B(Z) G(Z) K_{z}(Z)\right\}\right) .
\end{aligned}
$$

By (2.9) and the boundedness of $h_{\mu} \mathrm{s}$,

$$
K(z, z)=\sum_{\mu \in \mathbb{Z}} \frac{\left|h_{\mu}(z)\right|^{2}}{1+\lambda \gamma_{\mu}} \leq C \sum_{\mu \in \mathbb{Z}} \frac{1}{1+\lambda \gamma_{\mu}} \leq c \lambda^{-1 /(2 m)}=c h^{-1}
$$


where $c$ is a constant that does not rely on $z$. On the other hand,

$$
\begin{aligned}
& \left|E\left\{B(Z) G_{k}(Z) K_{z}(Z)\right\}\right|^{2} \\
= & \left|V\left(G_{k}, K_{z}\right)\right| \\
= & \left|\sum_{\mu} V\left(G_{k}, h_{\mu}\right) \frac{h_{\mu}(z)}{1+\lambda \gamma_{\mu}}\right|^{2} \\
\leq & \sum_{\mu}\left|V\left(G_{k}, h_{\mu}\right)\right|^{2} \sum_{\mu} \frac{\left|h_{\mu}(z)\right|^{2}}{\left(1+\lambda \gamma_{\mu}\right)^{2}} \\
\leq & c^{\prime} h^{-1}
\end{aligned}
$$

where $c^{\prime}$ is a constant that does not rely on $z$. So there exists a constant $c_{m}>0$ which does not rely on $u$ such that $\left\|R_{u}\right\| \leq c_{m} h^{-1 / 2}$. Then the conclusion of Lemma 2.4 holds.

S.6. A Concentration Inequality. The concentration inequality Lemma S.3 stated below will be used as a preliminary step in obtaining JBR.

Define $\mathcal{G}_{1}=\left\{g_{1}(x)=x^{T} \theta: x \in \mathbb{I}^{p},\left\|g_{1}\right\|_{\text {sup }} \leq 1, \theta \in \mathbb{R}^{p}\right\}$ and $\mathcal{G}_{2}=$ $\left\{g_{2}(z) \in H^{m}(\mathbb{I}):\left\|g_{2}\right\|_{\text {sup }} \leq 1, J\left(g_{2}, g_{2}\right) \leq c_{m}^{-2} h \lambda^{-1}\right\}$, where the constant $c_{m}$ is specified in Lemma 2.4. Let $\mathcal{G}=\mathcal{G}_{1}+\mathcal{G}_{2} \equiv\left\{g_{1}(x)+g_{2}(z): g_{1} \in\right.$ $\mathcal{G}_{1}$ and $\left.g_{2} \in \mathcal{G}_{2}\right\}$. For any $f \in \mathcal{G}$, define the empirical processes $Z_{n}(f)$ as

$$
Z_{n}(f)=\frac{1}{\sqrt{n}} \sum_{i=1}^{n}\left[\psi_{n}\left(T_{i} ; f\right) R_{U_{i}}-E_{T}\left(\psi_{n}(T ; f) R_{U}\right)\right]
$$

where $\psi_{n}(T ; f)$ is a real-valued function (possibly depending on $n$ ) defined on $\mathcal{T} \times \mathcal{G}$. Recall that $U_{i}=\left(X_{i}, Z_{i}\right)$ 's are covariates and $T_{i}=\left(Y_{i}, X_{i}, Z_{i}\right) \mathrm{s}$ denote the full data variables with domain $\mathcal{T}$.

Lemma S.3. Suppose that $\psi_{n}$ satisfies the following Lipschitz continuity:

$$
\left|\psi_{n}(T ; f)-\psi_{n}(T ; \tilde{f})\right| \leq c_{m}^{-1} h^{1 / 2}\|f-\tilde{f}\|_{\text {sup }} \text { for any } f, \tilde{f} \in \mathcal{G}
$$

where $c_{m}$ is specified in Lemma 2.4. Then we have

$$
\lim _{n \rightarrow \infty} P\left(\sup _{f \in \mathcal{G}} \frac{\left\|Z_{n}(f)\right\|}{h^{-(2 m-1) /(4 m)}\|f\|_{\text {sup }}^{1-1 /(2 m)}+n^{-1 / 2}} \leq(5 \log \log n)^{1 / 2}\right)=1 .
$$

We first establish and prove a preliminary lemma for proving Lemma S.3. Denote $N\left(\delta, \mathcal{G},\|\cdot\|_{\text {sup }}\right)$ as the $\delta$-covering number of the function class $\mathcal{G}$ in terms of the uniform norm. 
Lemma S.4. Suppose that $c_{m}^{-2} h \lambda^{-1}>1$. Then for any $\delta>0$,

$$
\log N\left(\delta, \mathcal{G},\|\cdot\|_{\text {sup }}\right) \leq C\left(h \lambda^{-1}\right)^{1 /(2 m)} \delta^{-1 / m},
$$

where $C>0$ is an universal constant.

Proof of Lemma S.4. By [18, Lemma 9.25],

$$
N\left(2 \delta, \mathcal{G},\|\cdot\|_{\text {sup }}\right) \leq N\left(\delta, \mathcal{G}_{1},\|\cdot\|_{\text {sup }}\right) N\left(\delta, \mathcal{G}_{2},\|\cdot\|_{\text {sup }}\right) .
$$

Since $N\left(\delta, \mathcal{G}_{1},\|\cdot\|_{\text {sup }}\right)$ is dominated by $N\left(\delta, \mathcal{G}_{2},\|\cdot\|_{\text {sup }}\right)$, it is sufficient to bound $N\left(\delta, \mathcal{G}_{2},\|\cdot\|_{\text {sup }}\right)$. Note that by $c_{m}^{-2} h \lambda^{-1}>1$,

$$
\begin{aligned}
\mathcal{G}_{2} & =\left(c_{m}^{-2} h \lambda^{-1}\right)^{1 / 2} \cdot\left\{g_{2} \in H^{m}(\mathbb{I}) \mid\left\|g_{2}\right\|_{\text {sup }} \leq\left(c_{m}^{-2} h \lambda^{-1}\right)^{-1 / 2}, J\left(g_{2}, g_{2}\right) \leq 1\right\} \\
& \subset\left(c_{m}^{-2} h \lambda^{-1}\right)^{1 / 2} \mathcal{T},
\end{aligned}
$$

where $\mathcal{T}=\left\{g \in H^{m}(\mathbb{I})\|g\|_{\text {sup }} \leq 1, J(g, g) \leq 1\right\}$. So by [34],

$$
\begin{aligned}
\log N\left(\delta, \mathcal{G}_{2},\|\cdot\|_{\text {sup }}\right) & \leq \log N\left(\delta,\left(c_{m}^{-2} h \lambda^{-1}\right)^{1 / 2} \mathcal{T},\|\cdot\|_{\text {sup }}\right) \\
& =\log N\left(\left(c_{m}^{-2} h \lambda^{-1}\right)^{-1 / 2} \delta, \mathcal{T},\|\cdot\|_{\text {sup }}\right) \\
& \leq c\left(\left(c_{m}^{-2} h \lambda^{-1}\right)^{-1 / 2} \delta\right)^{-1 / m} \\
& =c c_{m}^{-1 / m}\left(h \lambda^{-1}\right)^{1 /(2 m)} \delta^{-1 / m} .
\end{aligned}
$$

Now we prove Lemma S.3. For any $g, f \in \mathcal{G}$, by Lemma 2.4,

$$
\begin{aligned}
\left\|\left(\psi_{n}(T ; f)-\psi_{n}(T ; g)\right) R_{U}\right\| & \leq c_{m}^{-1} h^{1 / 2}\|f-g\|_{\text {sup }} \cdot\left\|R_{U}\right\| \\
& \leq c_{m}^{-1} h^{1 / 2}\|f-g\|_{\text {sup }} \cdot c_{m} h^{-1 / 2}=\|f-g\|_{\text {sup }}
\end{aligned}
$$

By Theorem 3.5 of [S2], for any $t>0$

$$
P\left(\left\|Z_{n}(f)-Z_{n}(g)\right\| \geq t\right) \leq 2 \exp \left(-\frac{t^{2}}{8\|f-g\|_{\text {sup }}^{2}}\right) .
$$

Then by Lemma 8.1 in [18], we have

$$
\|\| Z_{n}(g)-Z_{n}(f)\|\|_{\psi_{2}} \leq 8\|g-f\|_{\text {sup }}
$$

where $\|\cdot\|_{\psi_{2}}$ denotes the Orlicz norm associated with $\psi_{2}(s) \equiv \exp \left(s^{2}\right)-1$. Then it follows by Lemma S.4 and Theorem 8.4 of [18] that for arbitrary 
$\delta>0$,

$$
\begin{aligned}
& \left\|\sup _{\substack{g, f \in \mathcal{G} \\
\|g-f\|_{\sup } \leq \delta}}\right\| Z_{n}(g)-Z_{n}(f)\|\|_{\psi_{2}} \\
\leq & C^{\prime}\left(\int_{0}^{\delta} \sqrt{\log \left(1+N\left(\delta, \mathcal{G},\|\cdot\|_{\text {sup }}\right)\right)}+\delta \sqrt{\log \left(1+N\left(\delta, \mathcal{G},\|\cdot\|_{\text {sup }}\right)^{2}\right)}\right) \\
\asymp & h^{-(2 m-1) /(4 m)} \delta^{1-1 /(2 m)} .
\end{aligned}
$$

So, again, by Lemma 8.1 in [18],

$$
P\left(\sup _{\substack{g \in \mathcal{G} \\\|g\|_{\text {sup }} \leq \delta}}\left\|Z_{n}(g)\right\| \geq t\right) \leq 2 \exp \left(-h^{(2 m-1) /(2 m)} \delta^{-2+1 / m} t^{2}\right) .
$$

Let $b_{n}=n^{1 / 2} h^{-(2 m-1) /(4 m)}, \varepsilon=b_{n}^{-1}, \gamma=1-1 /(2 m), T_{n}=(5 \log \log n)^{1 / 2}$, and $Q_{\varepsilon}=\left[\gamma-\log _{2} \varepsilon-1\right]$, where $[a]$ denotes the integer part of $a$. Then by 
(S.7),

$$
\begin{aligned}
& P\left(\sup _{\substack{g \in \mathcal{G} \\
\|g\|_{\text {sup }} \leq 2}} \frac{\sqrt{n}\left\|Z_{n}(g)\right\|}{a_{n}\|g\|_{\text {sup }}^{\gamma}+1} \geq T_{n}\right) \\
& \leq P\left(\sup _{\substack{g \in \mathcal{G} \\
\|g\|_{\text {sup }} \leq \varepsilon^{1 / \gamma}}} \frac{\sqrt{n}\left\|Z_{n}(g)\right\|}{a_{n}\|g\|_{\text {sup }}^{\gamma}+1} \geq T_{n}\right) \\
& +\sum_{l=0}^{Q_{\varepsilon}} P\left(\sup _{\substack{g \in \mathcal{G} \\
\left(2^{l} \varepsilon\right)^{1 / \gamma} \leq\|g\|_{\sup } \leq\left(2^{l+1} \varepsilon\right)^{1 / \gamma}}} \frac{\sqrt{n}\left\|Z_{n}(g)\right\|}{a_{n}\|g\|_{\text {sup }}^{\gamma}+1} \geq T_{n}\right) \\
& \leq P\left(\sup _{\substack{g \in \mathcal{G} \\
\|g\|_{\sup } \leq \varepsilon^{1 / \gamma}}} \sqrt{n}\left\|Z_{n}(g)\right\| \geq T_{n}\right) \\
& +\sum_{l=0}^{Q_{\varepsilon}} P\left(\sup _{\substack{g \in \mathcal{G} \\
\|g\|_{\sup } \leq\left(2^{l+1} \varepsilon\right)^{1 / \gamma}}} \sqrt{n}\left\|Z_{n}(g)\right\| \geq\left(1+2^{l}\right) T_{n}\right) \\
& \leq 2 \exp \left(-h^{(2 m-1) /(2 m)}\left(\varepsilon^{1 / \gamma}\right)^{-2+1 / m} T_{n}^{2} / n\right) \\
& +\sum_{l=0}^{Q_{\varepsilon}} 2 \exp \left(-h^{(2 m-1) /(2 m)}\left[\left(2^{l+1} \varepsilon\right)^{1 / \gamma}\right]^{-2+1 / m} T_{n}^{2}\left(2^{l}+1\right)^{2} / n\right) \\
& =2 \exp \left(-T_{n}^{2}\right)+\sum_{l=0}^{Q_{\varepsilon}} 2 \exp \left(-2^{-2(l+1)} T_{n}^{2}\left(2^{l}+1\right)^{2}\right) \\
& \leq 2\left(Q_{\varepsilon}+2\right) \exp \left(-T^{2} / 4\right) \leq \mathrm{const} \cdot \log n(\log n)^{-5 / 4} \rightarrow 0,
\end{aligned}
$$

as $n \rightarrow \infty$. This proves the result.

S.7. Proof of Proposition 2.5. The proof proceeds in two steps. In Step I, we show that there exists an unique element $f_{\lambda} \in \mathbb{R}^{p} \times H^{m}(0,1)$ satisfying $S_{\lambda}\left(f_{\lambda}\right)=0$ and $\left\|f_{\lambda}-f_{0}\right\|=O\left(h^{m}\right)$. In Step II, we show that there exists an unique element $\widehat{f}_{n, \lambda} \in \mathbb{R}^{p} \times H^{m}(0,1)$ satisfying $S_{n, \lambda}\left(\widehat{f}_{n, \lambda}\right)=0$ and $\| \widehat{f}_{n, \lambda}-$ $f_{0} \|=O_{P}\left(r_{n}\right)$, where $r_{n}=(n h)^{-1 / 2}+h^{m}$. Recall that $\widehat{f}_{n, \lambda}$ is exactly the smoothing spline estimate.

Part I: Let $r_{1 n}=2\left(J\left(g_{0}, g_{0}\right)+1\right)^{1 / 2} h^{m}$, and define the operator

$$
T_{1 h}(f)=f+S_{\lambda}\left(f_{0}+f\right), f \in \mathbb{R}^{p} \times H^{m}(0,1) .
$$


It is easy to see that

$$
\left\|T_{1 h}(f)\right\| \leq\left\|f+S_{\lambda}\left(f_{0}+f\right)\right\|=\left\|f+S_{\lambda}\left(f_{0}+f\right)-S_{\lambda}\left(f_{0}\right)\right\|+\left\|S_{\lambda}\left(f_{0}\right)\right\| .
$$

Let $\mathbb{B}(\varepsilon)=\left\{f \in \mathbb{R}^{p} \times H^{m}(0,1):\|f\| \leq \varepsilon\right\}$ be the ball in $\mathbb{R}^{p} \times H^{m}(0,1)$ of radius $\varepsilon$. Since $r_{1 n}=o(1)$, for any $f \in \mathbb{B}\left(r_{1 n}\right)$ and $0 \leq s, s^{\prime} \leq 1$, the range of $f_{0}+s s^{\prime} f$ is contained in $\mathcal{I}$.

Note $S\left(f_{0}\right)=0$ which implies $S_{\lambda}\left(f_{0}\right)=-P_{\lambda} f_{0}$. It follows by equation (2.14) of the paper that

$$
\left\|S_{\lambda}\left(f_{0}\right)\right\|=\left\|P_{\lambda} f_{0}\right\| \leq \sqrt{\lambda J\left(g_{0}, g_{0}\right)} \leq\left(J\left(g_{0}, g_{0}\right)+1\right)^{1 / 2} h^{m}=r_{1 n} / 2 .
$$

On the other hand, by $D S_{\lambda}\left(f_{0}\right)=-i d$ (see Proposition 2.3), Assumption A1 (2.4) and $\left\|R_{u}\right\| \leq c_{m} h^{-1 / 2}$ for any $u \in[0,1]^{p} \times H^{m}(0,1)$, we get that

$$
\begin{aligned}
& \left\|f+S_{\lambda}\left(f_{0}+f\right)-S_{\lambda}\left(f_{0}\right)\right\| \\
= & \left\|f+D S_{\lambda}\left(f_{0}\right) f+\int_{0}^{1} \int_{0}^{1} s D^{2} S_{\lambda}\left(f_{0}+s s^{\prime} f\right) f f d s d s^{\prime}\right\| \\
= & \left\|\int_{0}^{1} \int_{0}^{1} s D^{2} S_{\lambda}\left(f_{0}+s s^{\prime} f\right) f f d s d s^{\prime}\right\| \\
= & \left\|\int_{0}^{1} \int_{0}^{1} s E\left\{\ell_{a}^{\prime \prime \prime}\left(Y ; X^{T}\left(\theta_{0}+s s^{\prime} \theta\right)+g_{0}(Z)+s s^{\prime} g(Z)\right)\left(X^{T} \theta+g(Z)\right)^{2} R_{U}\right\}\right\| \\
\leq & \int_{0}^{1} \int_{0}^{1} E\left\{E\left\{\mid \ell_{a}^{\prime \prime \prime}\left(Y ; X^{T}\left(\theta_{0}+s s^{\prime} \theta\right)+g_{0}(Z)+s s^{\prime} g(Z)\right) \| U\right\}\left(X^{T} \theta+g(Z)\right)^{2}\left\|R_{U}\right\|\right\} \\
\leq & C_{0} C_{1} C_{2} c_{m} h^{-1 / 2} E\left\{I(U)\left(X^{T} \theta+g(Z)\right)^{2}\right\} \leq C_{3} h^{-1 / 2}\|f\|^{2} \leq C_{3} h^{-1 / 2} r_{1 n}^{2},
\end{aligned}
$$

where $C_{3}=C_{0} C_{1} C_{2} c_{m}$ is absolute constant, and $C_{0}, C_{1}, C_{2}$ are specified in Assumption A1 (2.4). Therefore, as $n \rightarrow \infty,\left\|T_{1 h}(f)\right\| \leq C_{3} h^{-1 / 2} r_{1 n}^{2}+$ $r_{1 n} / 2<r_{1 n}$, where the last inequality follows by $C_{3} r_{1 n} h^{-1 / 2}=O\left(h^{m-1 / 2}\right)=$ $o(1)\left(\right.$ note $m>1 / 2$ and $h=o(1)$ lead to that $\left.h^{m-1 / 2}=o(1)\right)$. So $T_{1 h}\left(\mathbb{B}\left(r_{1 n}\right)\right) \subset$ $\mathbb{B}\left(r_{1 n}\right)$.

Next we show that $T_{1 h}$ is a contraction mapping. For any $f_{j}=\left(\theta_{j}, g_{j}\right) \in$ $\mathbb{B}\left(r_{1 n}\right)$ for $j=1,2$. Taylor's expansion leads to that

$$
\begin{aligned}
& T_{1 h}\left(f_{1}\right)-T_{1 h}\left(f_{2}\right) \\
= & f_{1}-f_{2}+S_{\lambda}\left(f_{0}+f_{1}\right)-S_{\lambda}\left(f_{0}+f_{2}\right) \\
= & \int_{0}^{1}\left[D S_{\lambda}\left(f_{0}+f_{2}+s\left(f_{1}-f_{2}\right)\right)-D S_{\lambda}\left(f_{0}\right)\right]\left(f_{1}-f_{2}\right) d s \\
= & \int_{0}^{1} \int_{0}^{1} s D^{2} S_{\lambda}\left(f_{0}+s^{\prime}\left(f_{2}+s\left(f_{1}-f_{2}\right)\right)\right)\left(f_{1}-f_{2}\right)\left(f_{2}+s\left(f_{1}-f_{2}\right)\right) d s d s^{\prime} .
\end{aligned}
$$


So by similar arguments as in the above analysis of $\left\|T_{1 h}(f)\right\|$ we get that

$$
\begin{aligned}
& \left\|T_{1 h}\left(f_{1}\right)-T_{1 h}\left(f_{2}\right)\right\| \\
\leq & \int_{0}^{1} \int_{0}^{1} E\left\{E\left\{\mid \ell_{a}^{\prime \prime \prime}\left(Y ;\left\langle R_{U}, f_{0}+s^{\prime}\left(f_{2}+s\left(f_{1}-f_{2}\right)\right)\right\rangle\right) \| U\right\}\right. \\
& \left.\left|\left\langle R_{U}, f_{1}-f_{2}\right\rangle\left\langle R_{U}, f_{2}+s\left(f_{1}-f_{2}\right)\right\rangle\right| \cdot\left\|R_{U}\right\|\right\} d s d s^{\prime} \\
\leq & C_{3} h^{-1 / 2}\left(\left\|f_{2}\right\|+\left\|f_{1}-f_{2}\right\|\right)\left\|f_{1}-f_{2}\right\| \leq 3 C_{3} h^{-1 / 2} r_{1 n}\left\|f_{1}-f_{2}\right\| \\
< & (1 / 2)\left\|f_{1}-f_{2}\right\|,
\end{aligned}
$$

where the last inequality is because $h^{-1 / 2} r_{1 n}=o(1)$ as $n \rightarrow \infty$.

Therefore, $T_{1 h}$ is a contraction mapping on $\mathbb{B}\left(r_{1 n}\right)$. By contraction mapping theorem [S3], there exists uniquely an element $f_{\lambda}^{\prime} \in \mathbb{B}\left(r_{1 n}\right)$ such that $T_{1 h}\left(f_{\lambda}^{\prime}\right)=f_{\lambda}^{\prime}$. Define $f_{\lambda}=f_{0}+f_{\lambda}^{\prime}$, then we have $S_{\lambda}\left(f_{\lambda}\right)=0$ and $\left\|f_{\lambda}-f_{0}\right\| \leq$ $r_{1 n}$.

Part II: Define the operator

$$
T_{2 h}(f)=f-\left[D S_{\lambda}\left(f_{\lambda}\right)\right]^{-1} S_{n, \lambda}\left(f_{\lambda}+f\right) .
$$

Here, the invertibility of $D S_{\lambda}\left(f_{\lambda}\right)$ follows by the below arguments: for all $f, f^{\prime} \in \mathbb{R}^{p} \times H^{m}(0,1)$,

$$
\begin{aligned}
& \left|\left[D S_{\lambda}\left(f_{\lambda}\right)-D S_{\lambda}\left(f_{0}\right)\right] f f^{\prime}\right| \\
= & \left|E\left\{\left(\ddot{\ell}_{a}\left(Y ;\left\langle R_{U}, f_{\lambda}\right\rangle\right)-\ddot{\ell}_{a}\left(Y ;\left\langle R_{U}, f_{0}\right\rangle\right)\right)\left\langle R_{U}, f\right\rangle\left\langle R_{U}, f^{\prime}\right\rangle\right\}\right| \\
\leq & \int_{0}^{1} E\left\{\left|\ell_{a}^{\prime \prime \prime}\left(Y ;\left\langle R_{U}, f_{0}+s\left(f_{\lambda}-f_{0}\right)\right\rangle\right)\right| \cdot\left|\left\langle R_{U}, f\right\rangle\right| \cdot\left|\left\langle R_{U}, f^{\prime}\right\rangle\right| \cdot\left|\left\langle R_{U}, f_{\lambda}-f_{0}\right\rangle\right|\right\} \\
\leq & C_{0} C_{1} C_{2} c_{m} h^{-1 / 2} r_{1 n}\|f\| \cdot\left\|f^{\prime}\right\| .
\end{aligned}
$$

Since $h^{-1 / 2} r_{1 n}=o(1)$, the above conclusion implies that the operator norm of the difference $D S_{\lambda}\left(f_{\lambda}\right)-D S_{\lambda}\left(f_{0}\right)$ is strictly less than $1 / 2$. Since $D S_{\lambda}\left(f_{0}\right)=$ $-i d, D S_{\lambda}\left(f_{\lambda}\right)$ is invertible. The norm of the inverse operator $D S_{\lambda}\left(f_{\lambda}\right)^{-1}$ is between $(1 / 2,3 / 2)$.

Rewrite $T_{2 h}$ as

$$
\begin{aligned}
T_{2 h}(f)= & -D S_{\lambda}\left(f_{\lambda}\right)^{-1}\left[D S_{n, \lambda}\left(f_{\lambda}\right) f-D S_{\lambda}\left(f_{\lambda}\right) f\right] \\
& -D S_{\lambda}\left(f_{\lambda}\right)^{-1}\left[S_{n, \lambda}\left(f_{\lambda}+f\right)-S_{n, \lambda}\left(f_{\lambda}\right)-D S_{n, \lambda}\left(f_{\lambda}\right) f\right] \\
& -D S_{\lambda}\left(f_{\lambda}\right)^{-1} S_{n, \lambda}\left(f_{\lambda}\right) .
\end{aligned}
$$

Denote the above three terms by $I_{1}, I_{2}, I_{3}$, respectively.

Next we handle the three terms and conclude the proof. To address $I_{3}$, for $i=1, \ldots, n$, define $O_{i}^{\prime}=\dot{\ell}_{a}\left(Y_{i} ;\left\langle R_{U_{i}}, f_{\lambda}\right\rangle\right) R_{U_{i}}$ and $O_{i}=O_{i}^{\prime}-E\left\{O_{i}^{\prime}\right\}$. Note 
that $S_{\lambda}\left(f_{\lambda}\right)=0$, and hence,

$$
\begin{aligned}
& E\left\{\left\|S_{n, \lambda}\left(f_{\lambda}\right)\right\|^{2}\right\} \\
= & E\left\{\left\|S_{n, \lambda}\left(f_{\lambda}\right)-S_{\lambda}\left(f_{\lambda}\right)\right\|^{2}\right\} \\
= & E\left\{\left\|\frac{1}{n} \sum_{i=1}^{n} O_{i}\right\|^{2}\right\} \\
= & n^{-1} E\left\{\left\|O_{i}\right\|^{2}\right\} \\
\leq & n^{-1} E\left\{\left|\dot{\ell}_{a}\left(Y ;\left\langle R_{U}, f_{\lambda}\right\rangle\right)\right|^{2}\left\|R_{U}\right\|^{2}\right\} \\
\leq & c_{m}^{2}(n h)^{-1} E\left\{\left|\dot{\ell}_{a}\left(Y ;\left\langle R_{U}, f_{\lambda}\right\rangle\right)\right|^{2}\right\} \\
\leq & 2 c_{m}^{2}(n h)^{-1} E\left\{\epsilon^{2}+\left|\dot{\ell}_{a}\left(Y ;\left\langle R_{U}, f_{\lambda}\right\rangle\right)-\dot{\ell}_{a}\left(Y ;\left\langle R_{U}, f_{0}\right\rangle\right)\right|^{2}\right\} \\
\leq & 2 c_{m}^{2}(n h)^{-1} E\left\{\epsilon^{2}+\sup _{a \in \mathcal{I}}\left|\ddot{\ell}_{a}(Y ; a)\right|^{2} \cdot\left|\left\langle R_{U}, f_{\lambda}-f_{0}\right\rangle\right|^{2}\right\} \\
\leq & 2 c_{m}^{2}(n h)^{-1}\left(E\left\{\epsilon^{2}\right\}+2 C_{1} C_{0}^{2} C_{2} E\left\{I(U)\left|\left\langle R_{U}, f_{\lambda}-f_{0}\right\rangle\right|^{2}\right\}\right) \\
\leq & 2 c_{m}^{2}(n h)^{-1}\left(E\left\{\epsilon^{2}\right\}+2 C_{1} C_{0}^{2} C_{2} r_{1 n}^{2}\right),
\end{aligned}
$$

where the last inequality follows by $E\left\{I(U)\left|\left\langle R_{U}, f_{\lambda}-f_{0}\right\rangle\right|^{2}\right\} \leq\left\|f_{\lambda}-f_{0}\right\|^{2} \leq$ $r_{1 n}^{2}$. Since $r_{1 n}=o(1)$, the above arguments imply that

$$
E\left\{\left\|S_{n, \lambda}\left(f_{\lambda}\right)\right\|^{2}\right\}=O\left((n h)^{-1}\right),
$$

therefore, $\left\|S_{n, \lambda}\left(f_{\lambda}\right)\right\|=O_{P}\left((n h)^{-1 / 2}\right)$. Let $C_{4}$ be large constant such that, with probability approaching one, $\left\|S_{n, \lambda}\left(f_{\lambda}\right)\right\| \leq C_{4}(n h)^{-1 / 2}$. Define $r_{2 n}=$ $2 C_{4}(n h)^{-1 / 2}$, and restrict $f$ to be an element in $\mathbb{B}\left(r_{2 n}\right) \equiv\left\{f \in \mathbb{R}^{p} \times\right.$ $\left.H^{m}(0,1):\|f\| \leq r_{2 n}\right\}$.

Second, we handle $I_{1}$. For $i=1,2, \ldots, n$, denote

$$
A_{i}=\left\{\sup _{a \in \mathcal{I}}\left|\ell_{a}^{\prime \prime \prime}\left(Y_{i} ; a\right)\right| \leq C \log n, \text { and } \sup _{a \in \mathcal{I}}\left|\ddot{\ell}_{a}\left(Y_{i} ; a\right)\right| \leq C \log n\right\} .
$$

By Assumption A1 (2.3)-(2.4) in the main paper, we may choose the above $C$ to be large so that $\cap_{i} A_{i}$ has probability approaching one, and $P\left(A_{i}^{c}\right)=$ $O\left(n^{-1}\right)$.

By Cauchy's inequality we have

$$
\begin{aligned}
& \left\|E\left\{\ddot{\ell}_{a}\left(Y ;\left\langle R_{U}, f_{\lambda}\right\rangle\right) I_{A^{c}}\left\langle R_{U}, f\right\rangle R_{U}\right\}\right\| \\
\leq & E\left\{\left|\ddot{\ell}_{a}\left(Y ;\left\langle R_{U}, f_{\lambda}\right\rangle\right)\right|^{2} I_{A^{c}}\left|\left\langle R_{U}, f\right\rangle\right|^{2}\left\|R_{U}\right\|^{2}\right\}^{1 / 2} \cdot P\left(A^{c}\right)^{1 / 2} \\
\leq & O\left((n h)^{-1 / 2}\right)\|f\|,
\end{aligned}
$$

where we dopped the subscript $i$ in the above derivations for simplicity. 
Without loss of generality, let $f=(\theta, g) \in \mathbb{B}\left(r_{2 n}\right) \backslash\{0\}$. Define $d_{n}=$ $c_{m}\|f\| h^{-1 / 2}$, and $\bar{f}=(\bar{\theta}, \bar{g}) \equiv f /\left(2 d_{n}\right)$. Then it follows that

$$
\|\bar{f}\|_{\text {sup }} \leq c_{m} h^{-1 / 2}\|\bar{f}\|=\left(\left(c_{m} h^{-1 / 2}\right) /\left(2 d_{n}\right)\right)\|f\|=1 / 2
$$

which implies that for any $(x, z) \in \mathcal{U},\left|x^{T} \bar{\theta}+\bar{g}(z)\right| \leq 1 / 2$. Letting $x$ approach zero, one gets that $|\bar{g}(z)| \leq 1 / 2$, and thus, $\|\bar{g}\|_{\text {sup }} \leq 1 / 2$, which further implies that $\left|x^{T} \bar{\theta}\right| \leq\|\bar{g}\|_{\text {sup }}+\|\bar{f}\|_{\text {sup }} \leq 1$ for any $x \in \mathbb{I}^{p}$. Also note that

$$
\begin{aligned}
J(\bar{g}, \bar{g}) & =d_{n}^{-2} \lambda^{-1}(\lambda J(g, g)) / 4 \\
& \leq d_{n}^{-2} \lambda^{-1}\|f\|^{2} / 4 \\
& \leq d_{n}^{-2} \lambda^{-1} r_{n}^{2} / 4 \\
& <c_{m}^{-2} h \lambda^{-1}
\end{aligned}
$$

Thus, $\bar{f}$ is an element in the class $\mathcal{G}$ of partial linear functions, where recall that $\mathcal{G}$ is defined in Section S.6 of the supplement document.

Let

$$
\psi\left(T_{i} ; \bar{f}\right)=\ddot{\ell}_{a}\left(Y_{i} ;\left\langle R_{U_{i}}, f_{\lambda}\right\rangle\right)\left\langle R_{U_{i}}, \bar{f}\right\rangle I_{A_{i}}
$$

and

$$
\psi_{n}\left(T_{i} ; \bar{f}\right)=(C \log n)^{-1} c_{m}^{-1} h^{1 / 2} \psi\left(T_{i} ; \bar{f}\right) .
$$

So for any $f_{1}, f_{2} \in \mathcal{G}$,

$$
\begin{aligned}
& \left|\psi_{n}\left(T_{i} ; f_{1}\right)-\psi_{n}\left(T_{i} ; f_{2}\right)\right| \\
= & (C \log n)^{-1} c_{m}^{-1} h^{1 / 2} I_{A_{i}}\left|\ddot{\ell}_{a}\left(Y_{i} ;\left\langle R_{U_{i}}, f_{\lambda}\right\rangle\right)\right| \cdot\left|\left\langle R_{U_{i}}, f_{1}-f_{2}\right\rangle\right| \\
\leq & (C \log n)^{-1} c_{m}^{-1} h^{1 / 2}(C \log n)\left\|f_{1}-f_{2}\right\|_{\text {sup }} \\
= & c_{m}^{-1} h^{1 / 2}\left\|f_{1}-f_{2}\right\|_{\text {sup }},
\end{aligned}
$$

implying that $\psi_{n}$ satisfies the Lipschitz continuity condition in Lemma S.3. Thus, with probability approaching one,

$$
\begin{aligned}
& \left\|\sum_{i=1}^{n}\left[\psi_{n}\left(T_{i} ; \bar{f}\right) R_{U_{i}}-E\left\{\psi_{n}\left(T_{i} ; \bar{f}\right) R_{U_{i}}\right\}\right]\right\| \\
\leq \quad & (5 \log \log n)^{1 / 2}\left(n^{1 / 2} h^{-(2 m-1) /(4 m)}+1\right),
\end{aligned}
$$

which, by $\bar{f}=f /\left(2 d_{n}\right)$, leads to that

$$
\begin{gathered}
\left\|\sum_{i=1}^{n}\left[\psi_{n}\left(T_{i} ; f\right) R_{U_{i}}-E\left\{\psi_{n}\left(T_{i} ; f\right) R_{U_{i}}\right\}\right]\right\| \\
\leq \quad 2 d_{n}(5 \log \log n)^{1 / 2}\left(n^{1 / 2} h^{-(2 m-1) /(4 m)}+1\right) .
\end{gathered}
$$


Therefore, on $\cap_{i} A_{i}$, we get that

$$
\begin{aligned}
& \left\|D S_{n, \lambda}\left(f_{\lambda}\right) f-D S_{\lambda}\left(f_{\lambda}\right) f\right\| \\
\leq & n^{-1} \| \sum_{i=1}^{n}\left[\ddot{\ell}_{a}\left(Y_{i} ;\left\langle R_{U_{i}}, f_{\lambda}\right\rangle\right)\left\langle R_{U_{i}}, f\right\rangle R_{U_{i}} I_{A_{i}}\right. \\
& \left.-E\left\{\ddot{\ell}_{a}\left(Y_{i} ;\left\langle R_{U_{i}}, f_{\lambda}\right\rangle\right)\left\langle R_{U_{i}}, f\right\rangle R_{U_{i}} I_{A_{i}}\right\}\right] \| \\
& +\left\|E\left\{\ddot{\ell}_{a}\left(Y_{i} ;\left\langle R_{U_{i}}, f_{\lambda}\right\rangle\right)\left\langle R_{U_{i}}, f\right\rangle R_{U_{i}} I_{A_{i}^{c}}\right\}\right\| \\
= & n^{-1}(C \log n) c_{m} h^{-1 / 2}\left\|\sum_{i=1}^{n}\left[\psi_{n}\left(T_{i} ; f\right) R_{U_{i}}-E\left\{\psi_{n}\left(T_{i} ; f\right) R_{U_{i}}\right\}\right]\right\| \\
& +O\left((n h)^{-1 / 2}\right)\|f\| \\
\leq & n^{-1}(C \log n) c_{m} h^{-1 / 2} 2 d_{n}(5 \log \log n)^{1 / 2}\left(n^{1 / 2} h^{-(2 m-1) /(4 m)}+1\right) \\
& +O\left((n h)^{-1 / 2}\right)\|f\| \\
= & O\left(n^{-1 / 2} h^{-(6 m-1) /(4 m)}(\log n)(\log \log n)^{1 / 2}+(n h)^{-1 / 2}\right)\|f\| \\
\leq & r_{2 n} / 8
\end{aligned}
$$

where the last equality follows by the trivial fact

$$
\begin{aligned}
(n h)^{-1 / 2} & \leq n^{-1 / 2} h^{-(6 m-1) /(4 m)}(\log n)(\log \log n)^{1 / 2} \\
& \leq n^{-1 / 2} h^{-2}(\log n)(\log \log n)^{1 / 2}=o(1)
\end{aligned}
$$

Therefore, with probability approaching one, for any $f \in \mathbb{B}\left(r_{2 n}\right),\left\|I_{1}\right\| \leq$ $\left\|D S_{\lambda}\left(f_{\lambda}\right)^{-1}\right\| \cdot\left\|D S_{n, \lambda}\left(f_{\lambda}\right) f-D S_{\lambda}\left(f_{\lambda}\right) f\right\| \leq 3 r_{2 n} / 16$.

Last, we address $I_{2}$ using similar arguments as in the analysis of $I_{1}$. It follows by Taylor's expansion that

$S_{n, \lambda}\left(f_{\lambda}+f\right)-S_{n, \lambda}\left(f_{\lambda}\right)-D S_{n, \lambda}\left(f_{\lambda}\right) f=\int_{0}^{1} \int_{0}^{1} s D^{2} S_{n, \lambda}\left(f_{\lambda}+s^{\prime} s f\right) f f d s d s^{\prime}$.

For any $s, s^{\prime} \in[0,1]$, let $\tilde{f}=f_{\lambda}+s^{\prime} s f$, then we have, on $\cap_{i} A_{i}$,

$$
\begin{aligned}
& \left\|D^{2} S_{n, \lambda}\left(f_{\lambda}+s^{\prime} s f\right) f f\right\| \\
= & n^{-1}\left\|\sum_{i=1}^{n} \ell_{a}^{\prime \prime \prime}\left(Y_{i} ;\left\langle R_{U_{i}}, \tilde{f}\right\rangle\right)\left|\left\langle R_{U_{i}}, f\right\rangle\right|^{2} R_{U_{i}}\right\| \\
\leq & n^{-1}(C \log n) c_{m} h^{-1 / 2} \sum_{i=1}^{n}\left|\left\langle R_{U_{i}}, f\right\rangle\right|^{2} \\
\leq & C c_{m}^{3}(\log n) h^{-3 / 2}\|f\|^{2} \leq C C_{4} c_{m}^{3}(\log n) n^{-1 / 2} h^{-2}\|f\| .
\end{aligned}
$$


Therefore, by $n^{-1 / 2} h^{-2}(\log n)=o(1)$,

$$
\left\|I_{2}\right\| \leq(3 / 2) C C_{4} c_{m}^{3}(\log n) n^{-1 / 2} h^{-2}\|f\|<r_{2 n} / 4 .
$$

By the above analysis of the terms $I_{1}, I_{2}, I_{3}$, we have that, with probability approaching one, for any $f \in \mathbb{B}\left(r_{2 n}\right)$,

$$
\left\|T_{2 h}(f)\right\| \leq\left\|I_{1}\right\|+\left\|I_{2}\right\|+\left\|I_{3}\right\| \leq 3 r_{2 n} / 16+r_{2 n} / 4+r_{2 n} / 2<r_{2 n}
$$

that is, $T_{2 n}\left(\mathbb{B}\left(r_{2 n}\right)\right) \subset \mathbb{B}\left(r_{2 n}\right)$.

To conclude Part II, we show that $T_{2 h}$ is a contraction mapping. For any $f_{1}, f_{2} \in \mathbb{B}\left(r_{2 n}\right)$, we have, by Taylor's expansion, that

$$
\begin{aligned}
& T_{2 h}\left(f_{1}\right)-T_{2 h}\left(f_{2}\right) \\
= & f_{1}-f_{2}-D S_{\lambda}\left(f_{\lambda}\right)^{-1}\left[S_{n, \lambda}\left(f_{\lambda}+f_{1}\right)-S_{n, \lambda}\left(f_{\lambda}+f_{2}\right)\right] \\
= & -D S_{\lambda}\left(f_{\lambda}\right)^{-1} \int_{0}^{1} \int_{0}^{1} D^{2} S_{n, \lambda}\left(f_{\lambda}+s^{\prime}\left(f_{2}+s\left(f_{1}-f_{2}\right)\right)\right) \\
& \left(f_{2}+s\left(f_{1}-f_{2}\right)\right)\left(f_{1}-f_{2}\right) d s d s^{\prime} \\
& -D S_{\lambda}\left(f_{\lambda}\right)^{-1}\left[D S_{n, \lambda}\left(f_{\lambda}\right)-D S_{\lambda}\left(f_{\lambda}\right)\right]\left(f_{1}-f_{2}\right) .
\end{aligned}
$$

Denote the two terms on the right hand side by $I_{4}, I_{5}$. Using exactly the same arguments as the analysis of the terms $I_{2}$ and $I_{1}$, it can be shown that, with probability approaching one, for any $f_{1}, f_{2} \in \mathbb{B}\left(r_{2 n}\right)$,

$$
\begin{gathered}
\left\|I_{4}\right\|=O\left(n^{-1 / 2} h^{-2}(\log n)\right)\left\|f_{1}-f_{2}\right\| \leq\left\|f_{1}-f_{2}\right\| / 3, \\
\left\|I_{5}\right\|=O\left(n^{-1 / 2} h^{-(6 m-1) /(4 m)}(\log n)(\log \log n)^{1 / 2}+(n h)^{-1 / 2}\right)\left\|f_{1}-f_{2}\right\| \\
\leq\left\|f_{1}-f_{2}\right\| / 3,
\end{gathered}
$$

implying that $\left\|T_{2 h}\left(f_{1}\right)-T_{2 h}\left(f_{2}\right)\right\| \leq 2\left\|f_{1}-f_{2}\right\| / 3$. Therefore, $T_{2 h}$ is a contraction mapping from $\mathbb{B}\left(r_{2 n}\right)$ to itself. By contraction mapping theorem, there exists uniquely an element $f^{\prime} \in \mathbb{B}\left(r_{2 n}\right)$ such that $T_{2 h}\left(f^{\prime}\right)=f^{\prime}$, implying $S_{n, \lambda}\left(f_{\lambda}+f^{\prime}\right)=0$. Let $\widehat{f}_{n, \lambda}=f_{\lambda}+f^{\prime}$, then $S_{n, \lambda}\left(\widehat{f}_{n, \lambda}\right)=0$, that is, $\widehat{f}_{n, \lambda}$ is the smoothing spline estimator. Furthermore, with probability approaching one,

$\left\|\widehat{f}_{n, \lambda}-f_{0}\right\| \leq\left\|\widehat{f}_{n, \lambda}-f_{\lambda}\right\|+\left\|f_{\lambda}-f_{0}\right\| \leq r_{2 n}+r_{1 n}=O\left((n h)^{-1 / 2}+h^{m}\right)=O\left(r_{n}\right)$.

This completes the proof. 
S.8. Proof of Theorem 2.6. The proof is rooted in [S4, 30].

By Assumption A1 (a), it is not difficult to check the following

$$
\max _{1 \leq i \leq n} \sup _{a \in \mathcal{I}}\left|\ddot{\ell}_{a}\left(Y_{i} ; a\right)\right|=O_{P}(\log n)
$$

So we can let $C>C_{0}$ be sufficiently large so that the event

$$
B_{n 1}=\left\{\max _{1 \leq i \leq n} \sup _{a \in \mathcal{I}}\left|\ddot{\ell}_{a}\left(Y_{i} ; a\right)\right| \leq C \log n\right\}
$$

has large probability.

Denote $f=\widehat{f}_{n, \lambda}-f_{0} \equiv(\theta, g)$. By Assumption A4, the event $B_{n 2}=\{\|f\| \leq$ $\left.r_{n} \equiv M\left((n h)^{-1 / 2}+h^{m}\right)\right\}$ has large probability with some preselected large $M$. So $B_{n}=B_{n 1} \cap B_{n 2}$ has large probability. Define $\bar{f} \equiv(\bar{\theta}, \bar{g})=d_{n}^{-1} f / 2$ (so $\bar{\theta}=d_{n}^{-1} \theta / 2$ and $\bar{g}=d_{n}^{-1} g / 2$ ), where $d_{n}=c_{m} r_{n} h^{-1 / 2}$. Since $h=o(1)$ and $n h^{2} \rightarrow \infty, d_{n}=o(1)$. Then by Lemma 2.4 , on $B_{n},\|\bar{f}\|_{\text {sup }} \leq 1 / 2$, which implies that for any $(x, z) \in \mathcal{U},\left|x^{T} \bar{\theta}+\bar{g}(z)\right| \leq 1 / 2$. Letting $x$ approach zero, one gets that $|\bar{g}(z)| \leq 1 / 2$, and thus, $\|\bar{g}\|_{\text {sup }} \leq 1 / 2$, which further implies that $\left|x^{T} \bar{\theta}\right| \leq\|\bar{g}\|_{\text {sup }}+\|\bar{f}\|_{\text {sup }} \leq 1$ for any $x \in \mathbb{I}^{p}$. Also note that

$$
\begin{aligned}
J(\bar{g}, \bar{g}) & =d_{n}^{-2} \lambda^{-1}(\lambda J(g, g)) / 4 \\
& \leq d_{n}^{-2} \lambda^{-1}\|f\|^{2} / 4 \\
& \leq d_{n}^{-2} \lambda^{-1} r_{n}^{2} / 4 \\
& <c_{m}^{-2} h \lambda^{-1} .
\end{aligned}
$$

Thus, when event $B_{n}$ holds, $\bar{f} \equiv f_{\tilde{\theta}, \tilde{g}}$ is an element in $\mathcal{G}$.

Define $\psi(T ; f)=\dot{\ell}_{a}\left(Y ; X^{T} \theta+g(Z)+X^{T} \theta_{0}+g_{0}(Z)\right)-\dot{\ell}_{a}\left(Y ; X^{T} \theta_{0}+g_{0}(Z)\right)$. By the definition of $S_{n, \lambda}$ and $S_{n}$, and a direct calculation, one can verify that

$S_{n}\left(f+f_{0}\right)-S\left(f+f_{0}\right)-\left(S_{n}\left(f_{0}\right)-S\left(f_{0}\right)\right)=\frac{1}{n} \sum_{i=1}^{n}\left[\psi\left(T_{i} ; f\right) R_{U_{i}}-E_{T}\left(\psi(T ; f) R_{U}\right)\right]$.

Let $\tilde{\psi}_{n}(T ; \bar{f})=(1 / 2) C^{-1} c_{m}^{-1}(\log n)_{.}^{-1} h^{1 / 2} d_{n}^{-1} \psi\left(T ; 2 d_{n} \bar{f}\right)$ and $\psi_{n}\left(T_{i} ; \bar{f}\right)=$ $\tilde{\psi}_{n}\left(T_{i} ; \bar{f}\right) I_{A_{i}}$, where $A_{i}=\left\{\sup _{a \in \mathcal{I}}\left|\ddot{\ell}_{a}\left(Y_{i} ; a\right)\right| \leq C \log n\right\}$ for $i=1, \ldots, n$. Observe that $B_{n}$ implies $\cap_{i} A_{i}$.

Next we show that $\psi_{n}$ satisfies (S.6) in the main paper. For any $\bar{f}_{1}=$ $\left(\theta_{1}, g_{1}\right), \bar{f}_{2}=\left(\theta_{2}, g_{2}\right) \in \mathcal{G}$, and $(x, z) \in \mathcal{U}$, since $x^{T} \theta_{0}+g_{0}(z) \in \mathcal{I}_{0}$ and $d_{n}=o(1)$, both $x^{T} \theta_{0}+g_{0}(z)+2 d_{n}\left(x^{T} \theta_{1}+g_{1}(z)\right)$ and $x^{T} \theta_{0}+g_{0}(z)+2 d_{n}\left(x^{T} \theta_{2}+\right.$ $\left.g_{2}(z)\right)$ fall in $\mathcal{I}$ when $n$ is sufficiently large (recall that $\mathcal{I}_{0}$ and $\mathcal{I}$ are specified 
in Assumption A1). Therefore,

$$
\begin{aligned}
& \left|\psi_{n}\left(T_{i} ; \bar{f}_{1}\right)-\psi_{n}\left(T_{i} ; \bar{f}_{2}\right)\right| \\
= & (1 / 2) C^{-1} c_{m}^{-1}(\log n)^{-1} h^{1 / 2} d_{n}^{-1}\left|\psi\left(T_{i} ; 2 d_{n} \bar{f}_{1}\right)-\psi\left(T_{i} ; 2 d_{n} \bar{f}_{2}\right)\right| \cdot I_{A_{i}} \\
= & (1 / 2) C^{-1} c_{m}^{-1}(\log n)^{-1} h^{1 / 2} d_{n}^{-1} \\
& \times \mid \int_{X_{i}^{T} \theta_{0}+g_{0}\left(Z_{i}\right)}^{X_{i}^{T} \theta_{0}+g_{0}\left(Z_{i}\right)+2 d_{n}\left(X^{T} \theta_{1}+g_{1}\left(Z_{i}\right)\right)} \ddot{\ell}_{a}\left(Y_{i} ; a\right) \cdot I_{A_{i}} d a \\
& -\int_{X_{i}^{T} \theta_{0}+g_{0}\left(Z_{i}\right)}^{X_{i}^{T} \theta_{0}+g_{0}\left(Z_{i}\right)+2 d_{n}\left(X_{i}^{T} \theta_{1}+g_{1}\left(Z_{i}\right)\right)} \ddot{\ell}_{a}\left(Y_{i} ; a\right) \cdot I_{A_{i}} d a \mid \\
\leq & C^{-1} c_{m}^{-1}(\log n)^{-1} h^{1 / 2} d_{n}^{-1} \cdot d_{n}\left\|\bar{f}_{1}-\bar{f}_{2}\right\|_{\sup } \cdot \sup _{a \in \mathcal{I}}\left|\ddot{\ell}_{a}\left(Y_{i} ; a\right)\right| \cdot I_{A_{i}} \\
\leq & C^{-1} c_{m}^{-1}(\log n)^{-1} h^{1 / 2} d_{n}^{-1} \cdot d_{n} \cdot C \log n \cdot\left\|\bar{f}_{1}-\bar{f}_{2}\right\|_{\sup } \\
= & c_{m}^{-1} h^{1 / 2}\left\|\bar{f}_{1}-\bar{f}_{2}\right\|_{\text {sup }} .
\end{aligned}
$$

Thus, $\psi_{n}$ satisfies (S.6). By Lemma S.3, on $B_{n}$ with large probability

$$
\begin{aligned}
&\left\|\sum_{i=1}^{n}\left[\psi_{n}\left(T_{i} ; \bar{f}\right) R_{U_{i}}-E_{T}\left\{\psi_{n}(T ; \bar{f}) R_{U}\right\}\right]\right\| \\
& \leq \quad\left(n^{1 / 2} h^{-(2 m-1) /(4 m)}+1\right)(5 \log \log n)^{1 / 2} .
\end{aligned}
$$

On the other hand, by Chebyshev's inequality

$$
P\left(A_{i}^{c}\right)=\exp \left(-\left(C / C_{0}\right) \log n\right) E\left\{\exp \left(\sup _{a \in \mathcal{I}}\left|\ddot{\ell}_{a}\left(Y_{i} ; a\right)\right| / C_{0}\right)\right\} \leq C_{1} n^{-C / C_{0}} .
$$

Since $h=o(1)$ and $n h^{2} \rightarrow \infty$, we may choose $C$ to be large so that $(\log n)^{-1} n^{-C /\left(2 C_{0}\right)}=o\left(a_{n}^{\prime} h^{1 / 2} d_{n}^{-1}\right)$, where recall that $a_{n}^{\prime}=n^{-1 / 2}\left((n h)^{-1 / 2}+\right.$ $\left.h^{m}\right) h^{-(6 m-1) /(4 m)}(\log \log n)^{1 / 2}$. By $(2.3)$ in the main paper, which implies $E\left\{\sup _{a \in \mathcal{I}}\left|\ddot{\ell}_{a}\left(Y_{i} ; a\right)\right| \mid U_{i}\right\} \leq 2 C_{1} C_{0}^{2}$, we have, on $B_{n}, E_{T}\left\{\left|\psi\left(T ; d_{n} g\right)\right|^{2}\right\} \leq$ $2 C_{1} C_{0}^{2} d_{n}^{2}$. So when $n$ is large, on $B_{n}$, by Chebyshev's inequality

$$
\begin{aligned}
& \left\|E_{T}\left\{\psi_{n}\left(T_{i} ; \bar{f}\right) R_{U_{i}}\right\}-E_{T}\left\{\tilde{\psi}_{n}\left(T_{i} ; \bar{f}\right) R_{U_{i}}\right\}\right\| \\
= & \left\|E_{T}\left\{\tilde{\psi}_{n}\left(T_{i} ; \bar{f}\right) R_{U_{i}} \cdot I_{A_{i}^{c}}\right\}\right\| \\
\leq & C^{-1}(\log n)^{-1} d_{n}^{-1}\left(E_{T}\left\{\left|\psi\left(T ; 2 d_{n} \bar{f}\right)\right|^{2}\right\}\right)^{1 / 2} P\left(A_{i}^{c}\right)^{1 / 2} \\
\leq & 2^{1 / 2} C^{-1} C_{0} C_{1}(\log n)^{-1} n^{-C /\left(2 C_{0}\right)} \\
\leq & M a_{n}^{\prime} h^{1 / 2} d_{n}^{-1} .
\end{aligned}
$$


Therefore, by (S.9) and on $B_{n}$,

$$
\begin{aligned}
& \left\|S_{n}\left(f+f_{0}\right)-S\left(f+f_{0}\right)-\left(S_{n}\left(f_{0}\right)-S\left(f_{0}\right)\right)\right\| \\
= & \frac{2 C c_{m}(\log n) h^{-1 / 2} d_{n}}{n}\left\|\sum_{i=1}^{n}\left[\tilde{\psi}_{n}\left(T_{i} ; \bar{f}\right) R_{U_{i}}-E_{T}\left\{\tilde{\psi}_{n}(T ; \bar{f}) R_{U}\right\}\right]\right\| \\
\leq & \frac{2 C c_{m}(\log n) h^{-1 / 2} d_{n}}{n}\left(\left\|\sum_{i=1}^{n}\left[\psi_{n}\left(T_{i} ; \bar{f}\right) R_{U_{i}}-E_{T}\left\{\psi_{n}(T ; \bar{f}) R_{U}\right\}\right]\right\|\right. \\
& \left.+n\left\|E_{T}\left\{\psi_{n}\left(T_{i} ; \bar{f}\right) R_{U_{i}}\right\}-E_{T}\left\{\tilde{\psi}_{n}\left(T_{i} ; \bar{f}\right) R_{U_{i}}\right\}\right\|\right) \\
\leq & \frac{2 C c_{m}(\log n) h^{-1 / 2} d_{n}}{n} \cdot\left[\left(n^{1 / 2} h^{-(2 m-1) /(4 m)}+1\right)(5 \log \log n)^{1 / 2}\right. \\
& \left.+n M a_{n}^{\prime} h^{1 / 2} d_{n}^{-1}\right] \leq C^{\prime} c_{m} a_{n}^{\prime} \log n,
\end{aligned}
$$

for some large constant $C^{\prime}>0$.

By Taylor's expansion, by the fact that $S_{n, \lambda}\left(f+f_{0}\right)=0$, and by Proposition 2.3,

$$
\begin{aligned}
& \left\|S_{n}\left(f+f_{0}\right)-S\left(f+f_{0}\right)-\left(S_{n}\left(f_{0}\right)-S\left(f_{0}\right)\right)\right\| \\
= & \left\|S_{n, \lambda}\left(f+f_{0}\right)-S_{\lambda}\left(f+f_{0}\right)-S_{n, \lambda}\left(f_{0}\right)+S_{\lambda}\left(f_{0}\right)\right\| \\
= & \left\|S_{\lambda}\left(f+f_{0}\right)+S_{n, \lambda}\left(f_{0}\right)-S_{\lambda}\left(f_{0}\right)\right\| \\
= & \left\|D S_{\lambda}\left(f_{0}\right) f+\int_{0}^{1} \int_{0}^{1} s D^{2} S_{\lambda}\left(f_{0}+s s^{\prime} f\right) f f d s d s^{\prime}+S_{n, \lambda}\left(f_{0}\right)\right\| \\
= & \left\|-f+\int_{0}^{1} \int_{0}^{1} s D^{2} S_{\lambda}\left(f_{0}+s s^{\prime} f\right) f f d s d s^{\prime}+S_{n, \lambda}\left(f_{0}\right)\right\| \\
\geq & \left\|-f+S_{n, \lambda}\left(f_{0}\right)\right\|-\left\|\int_{0}^{1} \int_{0}^{1} s D^{2} S_{\lambda}\left(f_{0}+s s^{\prime} f\right) f f d s d s^{\prime}\right\| .
\end{aligned}
$$

Therefore,

$$
\begin{aligned}
& \left\|f-S_{n, \lambda}\left(f_{0}\right)\right\| \\
\leq & \left\|S_{n}\left(f+f_{0}\right)-S\left(f+f_{0}\right)-\left(S_{n}\left(f_{0}\right)-S\left(f_{0}\right)\right)\right\| \\
& +\left\|\int_{0}^{1} \int_{0}^{1} s D^{2} S_{\lambda}\left(f_{0}+s s^{\prime} f\right) f f d s d s^{\prime}\right\| \\
\leq & \left\|S_{n}\left(f+f_{0}\right)-S\left(f+f_{0}\right)-\left(S_{n}\left(f_{0}\right)-S\left(f_{0}\right)\right)\right\| \\
& +\int_{0}^{1} \int_{0}^{1} s\left\|D^{2} S_{\lambda}\left(f_{0}+s s^{\prime} f\right) f f\right\| d s d s^{\prime} .
\end{aligned}
$$


Next we find an upper bound for $\left\|D^{2} S_{\lambda}\left(f_{0}+s s^{\prime} f\right) f f\right\|$. The Frechét derivative of $D S_{\lambda}$ is found to be $D^{2} S_{\lambda}=D^{2} S$, therefore,

$$
\begin{aligned}
& D^{2} S_{\lambda}\left(f_{0}+s s^{\prime} f\right) f f \\
= & D^{2} S\left(f_{0}+s s^{\prime} f\right) f f \\
= & E\left(\ell_{a}^{\prime \prime \prime}\left(Y ; X^{T}\left(\theta_{0}+s s^{\prime} \theta\right)+\left(g_{0}+s s^{\prime} g\right)(Z)\right)\left(X^{T} \theta+g(Z)\right)^{2} R_{U}\right) .
\end{aligned}
$$

Hence, by (2.4) in the main paper, on $B_{n}$,

$$
\begin{aligned}
& \left\|D^{2} S_{\lambda}\left(f_{0}+s s^{\prime} f\right) f f\right\| \\
= & \left\|E\left\{\ell_{a}^{\prime \prime \prime}\left(Y ; X^{T}\left(\theta_{0}+s s^{\prime} \theta\right)+\left(g_{0}+s s^{\prime} g\right)(Z)\right)\left(X^{T} \theta+g(Z)\right)^{2} R_{U}\right\}\right\| \\
\leq & E\left\{E\left\{\sup _{a \in \mathcal{I}} \mid \ell_{a}^{\prime \prime \prime}(Y ; a) \| U\right\}\left(X^{T} \theta+g(Z)\right)^{2}\left\|R_{U}\right\|\right\} \\
(\mathrm{S} .12) \leq & C_{\ell} c_{m} h^{-1 / 2}\|f\|^{2},
\end{aligned}
$$

where $C_{\ell}=\sup _{u \in \mathcal{U}} E\left\{\sup _{a \in \mathcal{I}}\left|\ell_{a}^{\prime \prime \prime}(Y ; a)\right| \mid U=u\right\}$. Thus, from (S.10), (S.11) and (S.12), with large probability

$$
\left\|f-S_{n, \lambda}\left(f_{0}\right)\right\| \leq C^{\prime} c_{m} a_{n}^{\prime} \log n+C_{\ell} c_{m} h^{-1 / 2}\left((n h)^{-1 / 2}+h^{m}\right)^{2} .
$$

This completes the proof of Theorem 2.6.

S.9. Proof of Corollary 3.2. We verify the conditions in Theorem 3.1 for Examples 5.1 and 5.2 respectively.

Example 5.1: In this situation, it is easy to see that $\ell(y ; a)=-(y-$ $a)^{2} /\left(2 \sigma^{2}\right)$. So $\dot{\ell}_{a}\left(Y ; X^{T} \theta_{0}+g_{0}(Z)\right)=\left(Y-X^{T} \theta_{0}-g_{0}(Z)\right) / \sigma^{2}, \ddot{\ell}_{a}(y ; a) \equiv-\sigma^{-2}$ and $\ell_{a}^{\prime \prime \prime}(y ; a) \equiv 0$, and hence, Assumption A1 holds. By calculations we have $B(Z)=\sigma^{-2}$. In this case, $V(g, \widetilde{g})$ becomes the usual $L^{2}$-norm. Assumption A2 holds when the basis functions are chosen as the eigenfunctions of the differential operator specified in $(2.10)$, or when $H_{0}^{m}(\mathbb{I})$ is used, the basis functions are chosen as the trigonometric functions.

By direct examinations, $h \asymp h^{*}$ satisfies all rate conditions required by Proposition 2.5 and Theorem 3.1 so that Assumption A4 holds. It can be shown by direct calculations that if $\sum_{\nu}\left|V\left(g_{0}, h_{\nu}\right)\right| \nu^{m}<\infty$, and $h \asymp h^{*}$, then as $n \rightarrow \infty$,

$$
\sqrt{n h}\left(W_{\lambda} g_{0}\right)\left(z_{0}\right)=\sqrt{n h^{2 m+1}} \sum_{\nu} V\left(g_{0}, h_{\nu}\right) \gamma_{\nu}^{1 / 2} \frac{\left(\lambda \gamma_{\nu}\right)^{1 / 2}}{1+\lambda \gamma_{\nu}} h_{\nu}\left(z_{0}\right) \rightarrow 0
$$

where the last limit follows by $\sup _{x \geq 0} \frac{x}{1+x^{2}}<\infty$ and dominated convergence theorem. The calculation of $\sigma_{z_{0}}^{2}$ follows by Remark 5.2 of [30]. Then the conclusion follows immediately from Theorem 3.1. 
Example 5.2: In this case we have $\ell(y ; a)=\alpha a+(\alpha-1) \log y-y \exp (a)$. Note that $\epsilon=\dot{\ell}_{a}\left(Y ; X^{T} \theta_{0}+g_{0}(Z)\right)=\alpha-Y \exp \left(X^{T} \theta_{0}+g_{0}(Z)\right)$, and $\ddot{\ell}_{a}(y ; a)=$ $\ell_{a}^{\prime \prime \prime}(y ; a)=-y \exp (a)$.

Since the components of $X$ are in $[0,1]$ and $g_{0}$ is bounded, there exists a constant $c>0$ such that $\left|X^{T} \theta_{0}+g_{0}(Z)\right| \leq c$, a.s. Therefore, $\mathcal{I}_{0}$, the range of the true semiparametric function $f_{0}(x, z) \equiv x^{T} \theta_{0}+g_{0}(z)$, is $[-c, c]$. Let $\mathcal{I}=[-2 c, 2 c]$, and choose constants $C_{0}$ and $C_{1}$ such that $C_{0}>\exp (3 c)$ and $C_{1}=\left(1-\exp (3 c) / C_{0}\right)^{-\alpha}$. Then we have

$$
\begin{aligned}
E\left\{\exp \left(\sup _{a \in \mathcal{I}}\left|\ddot{\ell}_{a}(Y ; a)\right| / C_{0}\right) \mid U\right\} & \leq E\left\{\exp \left(Y \exp (2 c) / C_{0}\right) \mid U\right\} \\
& =\left(1-\exp \left(2 c-X^{T} \theta_{0}-g_{0}(Z)\right) / C_{0}\right)^{-\alpha} \\
& \leq\left(1-\exp (3 c) / C_{0}\right)^{-\alpha}=C_{1} \text {, a.s. }
\end{aligned}
$$

$\ell_{a}^{\prime \prime \prime}(y ; a)$ satisfies the same property as in the above since $\ddot{\ell}_{a}(y ; a)=\ell_{a}^{\prime \prime \prime}(y ; a)$. It can also be directly shown that $E\left\{\epsilon^{4}\right\}=3 \alpha(2+\alpha)<\infty, E\left\{\epsilon^{2} \mid U\right\}=$ $I(U)=\alpha$, and thus $B(Z)=\alpha$ in this model. Then Assumption A1 holds. Meanwhile, we can construct the basis functions $h_{\nu}$ to satisfy Assumption A2 as those defined in (5.2) with $\sigma=\alpha^{-1 / 2}$, and the values of $\gamma_{\nu}$ as $\gamma_{0}=0$ and $\gamma_{2 k-1}=\gamma_{2 k}=\alpha^{-1}(2 \pi k)^{2 m}$ for $k \geq 1$. The calculations of $\sigma_{z_{0}}^{2}$ can be made in similar ways in Case (I) of Example 5.1, where properties of the trigonometric basis is used. Specifically, we get that $\sigma_{z_{0}}^{2}=I_{2} \alpha^{-(1-1 /(2 m))} / \pi$ and $c_{0}=I_{2} / I_{1}$, where $I_{1}, I_{2}$ are defined right below (5.3). It can be seen that $h \asymp h^{*}$ satisfies all rate conditions in Proposition 2.5 and Theorem 3.1, and therefore, Assumption A4 holds. Since the true parameter satisfies stronger smoothness conditions like $\sum_{\nu}\left|V\left(g_{0}, h_{\nu}\right)\right| \nu^{m}<\infty$, then it can be shown that $b_{z_{0}}=0$; see (S.13). Therefore, all the assumptions required by Theorems 3.1 are satisfied, so the conclusion of Corollary 3.2 holds.

S.10. Proof of Proposition 4.2. Denote the orthogonal complement of $\mathcal{H}_{0}$ and the subspace spanned by $\left\{R_{q_{j}, W_{j}}\right\}_{j=1}^{k}$ as $\mathcal{H}_{0}^{\perp}$ and $\operatorname{span}\left\{R_{q_{j}, W_{j}}\right\}_{j=1}^{k}$, respectively. We first show that

$$
\operatorname{span}\left\{R_{q_{j}, W_{j}}\right\}_{j=1}^{k}=\mathcal{H}_{0}^{\perp} .
$$

It is easy to see by (4.3) that $\operatorname{span}\left\{R_{q_{j}, W_{j}}\right\}_{j=1}^{k} \subset \mathcal{H}_{0}^{\perp}$. On the other hand, any element belonging to the orthogonal complement of $\operatorname{span}\left\{R_{q_{j}, W_{j}}\right\}_{j=1}^{k}$, which ought to be orthogonal to each $R_{q_{j}, W_{j}}$, is an element in $\mathcal{H}_{0}$. Therefore, $\mathcal{H}_{0}^{\perp} \subset \operatorname{span}\left\{R_{q_{j}, W_{j}}\right\}_{j=1}^{k}$. The above analysis implies (S.14).

Since $R_{u}-R_{u}^{0} \in \mathcal{H}_{0}^{\perp}$, we can write $R_{u}-R_{u}^{0}=\sum_{j=1}^{k} \rho_{u, j} R_{q_{j}, W_{j}}$. For simplicity, let $\rho_{u}=\left(\rho_{u, 1}, \ldots, \rho_{u, k}\right)^{T} \in \mathbb{R}^{k}$. Hence, it amounts to finding 
the form of $\rho_{u}$. Since $R_{u}-\sum_{j=1}^{k} \rho_{u, j} R_{q_{j}, W_{j}}=\left(H_{u}-\sum_{j=1}^{k} \rho_{u, j} H_{q_{j}, W_{j}}, T_{u}-\right.$ $\left.\sum_{j=1}^{k} \rho_{u, j} T_{q_{j}, W_{j}}\right)$ belongs to $\mathcal{H}_{0}$, we have $M\left(H_{u}-\sum_{j=1}^{k} \rho_{u, j} H_{q_{j}, W_{j}}\right)+Q\left(T_{u}\left(z_{0}\right)-\right.$ $\left.\sum_{j=1}^{k} \rho_{u, j} T_{q_{j}, W_{j}}\left(z_{0}\right)\right)=0$. Then, we have $M_{K} \rho_{u}=M H_{u}+Q T_{u}\left(z_{0}\right)$, which implies $\rho_{u}=M_{K}^{-1}\left(M H_{u}+Q T_{u}\left(z_{0}\right)\right)$. The invertibility of $M_{K}$ follows from the direct calculations below, based on (4.3),

$$
\begin{aligned}
M_{K} & =K\left(z_{0}, z_{0}\right) Q Q^{T}+\left(M-Q A\left(z_{0}\right)^{T}\right)\left(\Omega+\Sigma_{\lambda}\right)^{-1}\left(M-Q A\left(z_{0}\right)^{T}\right)^{T} \\
& =N\left(\begin{array}{cc}
I_{p} & 0 \\
-A\left(z_{0}\right)^{T} & 1
\end{array}\right)\left(\begin{array}{cc}
\left(\Omega+\Sigma_{\lambda}\right)^{-1} & 0 \\
0 & K\left(z_{0}, z_{0}\right)
\end{array}\right)\left(\begin{array}{cc}
I_{p} & -A\left(z_{0}\right) \\
0 & 1
\end{array}\right) N^{T},
\end{aligned}
$$

where $N$ is assumed to be full rank. With the above explicit $\rho_{u}$, we have obtained that $R_{u}^{0}=R_{u}-\sum_{j=1}^{k} \rho_{u, j} R_{q_{j}, W_{j}}$. As for $P_{\lambda}^{0}$, we can write $P_{\lambda}^{0} f=P_{\lambda} f-$ $\sum_{j=1}^{k} \zeta_{j}(f) R_{q_{j}, W_{j}}$ based on (S.14), for some $\zeta(f) \equiv\left(\zeta_{1}(f), \ldots, \zeta_{k}(f)\right)^{T} \in \mathbb{R}^{k}$. Similar arguments as above give that $\zeta(f)=M_{K}^{-1}\left(M H_{g}^{*}+Q T_{g}^{*}\left(z_{0}\right)\right)$.

S.11. Proof of Theorem 4.3. The proof is similar to those in Theorem 2.6, so we only sketch the idea. Let $f=\widehat{f}_{n, \lambda}^{0}-f_{0}^{0}$. Assumption A5 guarantees that with large probability, $\|f\| \leq r_{n} \equiv M\left((n h)^{-1 / 2}+h^{m}\right)$ for a proper large $M$. By a modification of the proof of Lemma S.3, we have the following lemma.

Lemma S.5. Suppose that $\psi_{n}$ satisfies Lipschitz continuity, namely, there exists a constant $C_{\psi}>0$ such that

$$
\left|\psi_{n}\left(T ; f_{1}\right)-\psi_{n}\left(T ; f_{2}\right)\right| \leq c_{m}^{-1} h^{1 / 2}\left\|f_{1}-f_{2}\right\|_{\text {sup }} \text {, for all } f_{1}, f_{2} \in \mathcal{H}_{0}
$$

where recall that $T=(Y, X, Z)$ denotes the full data variable. Then we have

$\lim _{n \rightarrow \infty} P\left(\sup _{\substack{f \in \mathcal{G}_{0} \\\|f\|_{\text {sup }} \leq 1}} \frac{\left\|Z_{n}^{0}(f)\right\|}{n^{1 / 2} h^{-(2 m-1) /(4 m)}\|f\|_{\text {sup }}^{1-1 /(2 m)}+1} \leq(5 \log \log n)^{1 / 2}\right)=1$,

where $\mathcal{G}_{0}=\mathcal{H}_{0} \cap \mathcal{G}$, and $Z_{n}^{0}(f)=\sum_{i=1}^{n}\left[\psi_{n}\left(T_{i} ; f\right) R_{U_{i}}^{0}-E_{T}\left\{\psi_{n}(T ; f) R_{U}^{0}\right\}\right]$.

By a reexamination of the proof of Theorem 2.6, we have, with large probability, $f=(\theta, g) \in \mathcal{G}_{0}$ and $\psi_{n}$ satisfies Lipschitz continuity (S.15), where

$$
\begin{aligned}
& \psi_{n}(T ; f) \\
= & C^{-1} c_{m}^{-1}(\log n)^{-1} h^{1 / 2} d_{n}^{-1}\left\{\dot{\ell}_{a}\left(Y ; X^{T}\left(\theta_{0}^{0}+d_{n} \theta\right)+g_{0}^{0}(Z)+d_{n} g(Z)\right)\right. \\
& \left.-\dot{\ell}_{a}\left(Y ; X^{T} \theta_{0}^{0}+g_{0}^{0}(Z)\right)\right\}
\end{aligned}
$$


and $d_{n}=c_{m} r_{n} h^{-1 / 2}$. This leads to, with large probability,

$$
\begin{aligned}
&\left\|\sum_{i=1}^{n}\left[\psi_{n}\left(T_{i} ; g\right) R_{U_{i}}^{0}-E_{T}\left\{\psi_{n}(T ; f) R_{U}^{0}\right\}\right]\right\|_{1} \\
& \leq \quad\left(n^{1 / 2} h^{-(2 m-1) /(4 m)}+1\right)(5 \log \log n)^{1 / 2} .
\end{aligned}
$$

The remainder of the proof follows by (S.8), and by an argument similar to (S.10) through (S.12).

S.12. Proof of Corollary 4.5. By Fourier expansion of $g_{0}$ and $W_{\lambda} h_{\nu}=$ $\frac{\lambda \gamma_{\nu}}{1+\lambda \gamma_{\nu}}$, we have $\left(W_{\lambda} g_{0}\right)\left(z_{0}\right)=\sum_{\nu} V\left(g_{0}, h_{\nu}\right) \frac{\lambda \gamma_{\nu}}{1+\lambda \gamma_{\nu}} h_{\nu}\left(z_{0}\right)$. By the assumption that $\sum_{\nu}\left|V\left(g_{0}, h_{\nu}\right)\right|^{2} \gamma_{\nu}^{d}<\infty$, one obtains the bound $\left|\left(W_{\lambda} g_{0}\right)\left(z_{0}\right)\right|=$ $O\left(\left(\lambda^{d} h^{-1}\right)^{1 / 2}\right)=O\left(h^{m d-1 / 2}\right)$ by using Cauchy's inequality. Thus, by $h \asymp$ $n^{-1 /(2 m+1)}$ and $d>1+1 /(2 m),(n h)^{1 / 2}\left(W_{\lambda} g_{0}\right)\left(z_{0}\right)=o(1)$. Direct calculations can verify that $h=o(1), n h^{2} \rightarrow \infty, a_{n}=o\left((n h)^{-1 / 2}+h^{m}\right)$, $a_{n}=o\left(n^{-1 / 2} h^{1 / 2}(\log n)^{-1}\right), a_{n}=o\left(n^{-1}\left((n h)^{-1 / 2}+h^{m}\right)^{-1}(\log n)^{-1}\right), a_{n} \gg$ $\left((n h)^{-1 / 2}+h^{m}\right)^{2} h^{-1 / 2}$, and $n^{1 / 2} h^{m(1+b)}=o(1)$. Thus, the desired result follows from Theorem 4.3.

S.13. Proof of Corollary 4.7. By similar arguments in the proof of Corollary 3.2, we get that all the assumptions in Corollary 4.5 are satisfied. To conclude the proof, we only prove the formula for $c_{0}$. Note $B(z) \equiv \sigma^{-2}$, where $\sigma^{2}$ is the error variance in partial smoothing spline model (see Example 5.1), and the reciprocal of the shape parameter in shape-rate Gamma model (see Example 5.2).

Let $\left(\widetilde{h}_{\nu}, \widetilde{\gamma}_{\nu}\right)$ be the eigenfunctions and eigenvalues of the following problem:

$(-1)^{m} \widetilde{h}_{\nu}^{(2 m)}(\cdot)=\tilde{\gamma}_{\nu} \pi(\cdot) \widetilde{h}_{\nu}(\cdot), \widetilde{h}_{\nu}^{(j)}(0)=\widetilde{h}_{\nu}^{(j)}(1)=0, j=m, m+1, \ldots, 2 m-1$.

Moreover, $\widetilde{h}_{\nu}$ is normalized such that $\int_{0}^{1} \pi(z) \widetilde{h}_{\nu}(z)^{2} d z=1$ for any $\nu \geq 1$. Define $\lambda^{\dagger}=\sigma^{2} \lambda, h^{\dagger}=\left(\lambda^{\dagger}\right)^{1 /(2 m)}, h_{\nu}=\sigma \tilde{h}_{\nu}$ and $\gamma_{\nu}=\sigma^{2} \widetilde{\gamma}_{\nu}$. Then it can be directly verified that $\left(h_{\nu}, \gamma_{\nu}\right)$ are the eigenfunctions and eigenvalues of the problem (2.10).

The kernel function $\widetilde{K}(s, t) \equiv \sum_{\nu \geq 1} \frac{\widetilde{h}_{\nu}(s) \widetilde{h}_{\nu}(t)}{1+\lambda^{\dagger} \widetilde{\gamma}_{\nu}}$ is the reproducing kernel with respect to the inner product $\left\langle g_{1}, g_{2}\right\rangle_{*}=\int_{0}^{1} \pi(z) g_{1}(z) g_{2}(z) d z+$ $\lambda^{\dagger} \int_{0}^{1} g_{1}^{(m)}(z) g_{2}^{(m)}(z) d z$. To see this, for any $g=\sum_{\nu} g_{\nu} \widetilde{h}_{\nu}$, it can be directly examined that $\langle\widetilde{K}(z, \cdot), g\rangle_{*}=\sum_{\nu} g_{\nu} \widetilde{h}_{\nu}(z)$, for all $z \in \mathbb{I}$. It can be also observed that $K(s, t)=\sum_{\nu \geq 1} \frac{h_{\nu}(s) h_{\nu}(t)}{1+\lambda \gamma_{\nu}}=\sigma^{2} \sum_{\nu \geq 1} \frac{\widetilde{h}_{\nu}(s) \widetilde{h}_{\nu}(t)}{1+\lambda^{\dagger} \widetilde{\gamma}_{\nu}}=\sigma^{2} \widetilde{K}(s, t)$. 
It follows by $[22,26]$ that there exists a square integrable function $\omega_{0}$ (with domain $\mathbb{R}$ ) such that for any $\varphi \in(0,1)$ and $s \in\left(\left(h^{\dagger}\right)^{\varphi}, 1-\left(h^{\dagger}\right)^{\varphi}\right)$,

$$
\sup _{t \in \mathbb{I}}\left|\widetilde{K}(s, t)-\left(h^{\dagger}\right)^{-1} \omega_{0}\left((s-t) / h^{\dagger}\right)\right|=O\left(\left(h^{\dagger}\right)^{-1} \exp \left(-c^{\prime}\left(h^{\dagger}\right)^{-1+\varphi}\right)\right),
$$

where $c^{\prime}>0$ is some absolute constant. Therefore,

$$
\sup _{t \in \mathbb{I}}\left|K(s, t)-\sigma^{2-1 / m} h^{-1} \omega_{0}\left(\sigma^{-1 / m}(s-t) / h\right)\right|=O\left(h^{-1} \exp \left(-c h^{-1+\varphi}\right)\right),
$$

for some absolute constant $c>0$. Then we have that, by square integrability of $\omega_{0}$ and dominated convergence theorem,

$$
\begin{aligned}
& E\left\{B(Z)\left|K_{z_{0}}(Z)\right|^{2}\right\} \\
= & \sigma^{-2} \int_{0}^{1} \pi(z)\left|K\left(z, z_{0}\right)\right|^{2} d z \\
= & \sigma^{-2} \int_{0}^{1} \pi(z)\left(\sigma^{2-1 / m} h^{-1} \omega_{0}\left(\sigma^{-1 / m}\left(z-z_{0}\right) / h\right)\right)^{2} d z+O\left(h^{-2} \exp \left(-c h^{-1+\varphi}\right)\right) \\
= & \sigma^{2-2 / m} h^{-2} \int_{0}^{1} \pi(z) \omega_{0}\left(\sigma^{-1 / m}\left(z-z_{0}\right) / h\right)^{2} d z+O\left(h^{-2} \exp \left(-c h^{-1+\varphi}\right)\right) \\
= & \sigma^{2-1 / m} h^{-1} \int_{-\frac{z_{0}}{\sigma^{1 / m} h}}^{\frac{1-z_{0}}{\sigma^{1 / m}}} \pi\left(z_{0}+\sigma^{1 / m} h l\right) \omega_{0}(l)^{2} d l+O\left(h^{-2} \exp \left(-c h^{-1+\varphi}\right)\right) \\
= & \sigma^{2-1 / m} h^{-1} \pi\left(z_{0}\right) \int_{\mathbb{R}} \omega_{0}(t)^{2} d t+o\left(h^{-1}\right) .
\end{aligned}
$$

On the other hand, $K\left(z_{0}, z_{0}\right)=\sigma^{2-1 / m} h^{-1} \omega_{0}(0)+o\left(h^{-1}\right)$. Therefore,

$$
c_{0}=\lim _{h \rightarrow 0} \frac{E\left\{B(Z)\left|K_{z_{0}}(Z)\right|^{2}\right\}}{K\left(z_{0}, z_{0}\right)}=\frac{\pi\left(z_{0}\right) \int_{\mathbb{R}} \omega_{0}(t)^{2} d t}{\omega_{0}(0)},
$$

where the limit on right side is free of the true parameters and the nuisance parameter $\sigma^{2}$.

When $m=2(3)$, the form of $\omega_{0}$ is explicitly given in [22]. So, if $\pi(z)$ is uniform, then we can explicitly find the value of $c_{0}$ which is $0.75(0.83)$.

S.14. Asymptotic Variance of the Parametric Estimator. In this section we will show that, in quasi-likelihood setup, the information bound derived for our parametric estimator is actually identical to the one derived by [23].

Proposition S.1. In one dimensional setup, i.e., $p=1$, the information bound derived by [23] in their quasi-likelihood setup is the same as $\Omega^{-1}$ in our setup. 
Proof of Proposition S.1. In the quasi-likelihood setup, $\ell(y ; a)=$ $Q(y ; F(a))$, where $F$ is the link function such that

$$
E\{Y \mid X, Z\}=F\left(X \theta_{0}+g_{0}(Z)\right)
$$

and $Q(y ; \mu)=\int_{y}^{\mu} \frac{y-s}{\mathcal{V}(s)} d s$, and $\mathcal{V}$ is the variance function. In this setup, it can be shown by direct calculations that $\dot{\ell}_{a}(y ; a)=(y-F(a)) \dot{F}(a) / \mathcal{V}(F(a))$, and $\ddot{\ell}_{a}(Y ; a)=F_{1}(a)+\varepsilon F_{2}(a)$, where $\varepsilon=Y-\mu_{0}(Z), F_{1}(a)=-|\dot{F}(a)|^{2} / \mathcal{V}(F(a))+$ $\left(F\left(g_{0}(Z)\right)-F(a)\right) F_{2}(a)$, and $F_{2}(a)=\left(\ddot{F}(a) \mathcal{V}(F(a))-\dot{\mathcal{V}}(F(a))|\dot{F}(a)|^{2}\right) / \mathcal{V}^{2}(F(a))$. So, $I(U)=\frac{\left|\dot{F}\left(X \theta_{0}+g_{0}(Z)\right)\right|^{2}}{\mathcal{V}\left(X \theta_{0}+g_{0}(Z)\right)}$.

[23] showed that their parametric estimator $\widehat{\theta}$ satisfies

$$
\sqrt{n}\left(\widehat{\theta}-\theta_{0}\right)=\frac{n^{-1 / 2} \sum_{i=1}^{n} W_{i} \frac{\dot{F}\left(X_{i} \theta_{0}+g_{0}\left(Z_{i}\right)\right)}{\mathcal{V}\left(F\left(X_{i} \theta_{0}+g_{0}\left(Z_{i}\right)\right)\right)}\left(X_{i}-h_{1}\left(Z_{i}\right)\right)}{E\left\{\frac{\left|\dot{F}\left(X \theta_{0}+g_{0}(Z)\right)\right|^{2}}{\mathcal{V}\left(X \theta_{0}+g_{0}(Z)\right)}\left(X-h_{1}(Z)\right)^{2}\right\}}+o_{P}(1)
$$

where

$$
\begin{aligned}
& h_{1}(Z) \\
= & \frac{E\left\{X\left|\dot{F}\left(X \theta_{0}+g_{0}(Z)\right)\right|^{2} / \mathcal{V}\left(X \theta_{0}+g_{0}(Z)\right) \mid Z\right\}}{E\left\{\left|\dot{F}\left(X \theta_{0}+g_{0}(Z)\right)\right|^{2} / \mathcal{V}\left(X \theta_{0}+g_{0}(Z)\right) \mid Z\right\}} \\
= & \frac{E\{X I(U) \mid Z\}}{E\{I(U) \mid Z\}}=G(Z),
\end{aligned}
$$

and $W_{i}=Y_{i}-F\left(X_{i} \theta_{0}+g_{0}\left(Z_{i}\right)\right)$. Note that by our notation we get

$$
\begin{aligned}
& \epsilon_{i} \\
= & \dot{\ell}_{a}\left(Y_{i} ; X_{i} \theta_{0}+g_{0}\left(Z_{i}\right)\right) \\
= & \left(Y_{i}-F\left(X_{i} \theta_{0}+g_{0}\left(Z_{i}\right)\right)\right) \dot{F}\left(X_{i} \theta_{0}+g_{0}\left(Z_{i}\right)\right) / \mathcal{V}\left(F\left(X_{i} \theta_{0}+g_{0}\left(Z_{i}\right)\right)\right)
\end{aligned}
$$

therefore, (S.17) becomes

$$
\sqrt{n}\left(\widehat{\theta}-\theta_{0}\right)=\frac{n^{-1 / 2} \sum_{i=1}^{n} \epsilon_{i}\left(X_{i}-G\left(Z_{i}\right)\right)}{\Omega}+o_{P}(1) \stackrel{d}{\rightarrow} N\left(0, \Omega^{-1}\right),
$$

where $\Omega=E\left\{I(U)(X-G(Z))^{2}\right\}$, the same as the information bound of the parametric estimator derived in our paper.

S.15. Technical Details of Example 5.3. In this section, we will demonstrate reasonable situations where the conditions in Theorems 3.1 and 4.4 are satisfied in Example 5.3. That is, the following proposition holds. 
Proposition S.2. Let the following conditions hold:

(1) The conditional probability density function of $X$ given $Z$, namely, $\pi(x \mid z)$, has bounded lth order partial derivatives with respect to $z$, for $l \leq m$.

(2) For any constant vector $v \neq 0, P\left(v^{T}(X-G(Z))=0\right)<1$.

(3) $g_{0}$ is sufficiently smooth which satisfies the conditions in Corollary 4.5.

(4) $m>1+\sqrt{3} / 2$ and $h \asymp h^{*}$.

Then the results of Theorems 3.1 and 4.4 hold in Example 5.3 for $b=1$.

Proof of Proposition S.2. In the logistic regression example, it is easy to see that $\ell(y ; a)=a y-\log (1+\exp (a))$. Then we have $\dot{\ell}_{a}(y ; a)=$ $y-\exp (a) /(1+\exp (a)), \ddot{\ell}_{a}(y ; a)=-\exp (a) /(1+\exp (a))^{2}$, and $\ell_{a}^{\prime \prime \prime}(y ; a)=$ $-\exp (a)(1-\exp (a)) /(1+\exp (a))^{3}$. Thus, $\epsilon=Y-\exp \left(X^{T} \theta_{0}+g_{0}(Z)\right) /(1+$ $\left.\exp \left(X^{T} \theta_{0}+g_{0}(Z)\right)\right)$. Note that both $\ddot{\ell}_{a}(y ; a)$ and $\ell_{a}^{\prime \prime \prime}(y ; a)$ are bounded, so Assumption A1 (2.3)-(2.4) are satisfied. A straightforward calculation gives

$$
\begin{aligned}
& E\left\{\epsilon^{2} \mid U\right\}=I(U)=\frac{\exp \left(X^{T} \theta_{0}+g_{0}(Z)\right)}{\left(1+\exp \left(X^{T} \theta_{0}+g_{0}(Z)\right)\right)^{2}}, \text { and } \\
& B(Z)=E\left\{\frac{\exp \left(X^{T} \theta_{0}+g_{0}(Z)\right)}{\left(1+\exp \left(X^{T} \theta_{0}+g_{0}(Z)\right)\right)^{2}} \mid Z\right\} .
\end{aligned}
$$

Since $X^{T} \theta_{0}+g_{0}(Z)$ is almost surely bounded, $I(U)$ has positive lower bound and finite upper bound, i.e., Assumption A1 (b) holds. Since $\epsilon$ is almost surely bounded, Assumption A1 (c) holds.

The solution $\gamma_{\nu}$ and $h_{\nu}$ of the problem (2.10) satisfy Assumption A2. Recall that

$G(Z)=E\{I(U) X \mid Z\} / B(Z)=E\left\{\frac{\exp \left(X^{T} \theta_{0}+g_{0}(Z)\right)}{\left(1+\exp \left(X^{T} \theta_{0}+g_{0}(Z)\right)\right)^{2}} X \mid Z\right\} / B(Z)$.

Although the numerator and denominator both have intractable forms, they can be sufficiently smooth so that $G$ can satisfy (3.1). For instance, when $X$ and $Z$ are independent, $B(z)=\int_{\mathbb{I}^{p}} \frac{\exp \left(x^{T} \theta_{0}+g_{0}(z)\right)}{\left(1+\exp \left(x^{T} \theta_{0}+g_{0}(z)\right)\right)^{2}} \pi_{1}(x) d x$, and $E\{I(U) X \mid Z=$ $z\}=\int_{\mathbb{I}^{p}} \frac{\exp \left(x^{T} \theta_{0}+g_{0}(z)\right)}{\left(1+\exp \left(x^{T} \theta_{0}+g_{0}(z)\right)\right)^{2}} x \pi_{1}(x) d x$, where $\pi_{1}(x)$ denotes the marginal density of $X$. Then, $E\{I(U) X \mid Z=z\}$ and $B(z)$ both belong to $H^{m}(\mathbb{I})$. We only illustrate this for $B(z)$ since the illustration is similar for $E\{I(U) X \mid Z=z\}$. Let $H(u)=\exp (u) /(1+\exp (u))^{2}$. Then $B(z)=\int_{\mathbb{I} p} H\left(x^{T} \theta_{0}+g_{0}(z)\right) \pi_{1}(x) d x$. It follows by Faá di Bruno's formula ([S1]) that the $m$-order derivative of $B(z)$ is a finite sum of terms like

$$
\text { constant } \times \int_{\mathbb{I}^{p}} H^{\left(n_{1}+\cdots+n_{m}\right)}\left(x^{T} \theta_{0}+g_{0}(z)\right) \prod_{l=1}^{m}\left[g_{0}^{(l)}(z)\right]^{n_{l}} \pi_{1}(x) d x,
$$


where $n_{1}, \ldots, n_{m}$ are nonnegative integers such that $\sum_{l=1}^{m} l n_{l}=m$. Since $H$ has bounded derivatives of arbitrary orders, and for $l \leq m-1,\left|g_{0}^{(l)}\left(z_{0}\right)\right|$ is bounded, there is an absolute constant $C^{\prime}$ such that

$$
\left|\int_{\mathbb{I}^{p}} H^{\left(n_{1}+\cdots+n_{m}\right)}\left(x^{T} \theta_{0}+g_{0}(z)\right) \prod_{l=1}^{m}\left[g_{0}^{(l)}(z)\right]^{n_{l}} \pi_{1}(x) d x\right| \leq C^{\prime}\left|g_{0}^{(m)}(z)\right|^{n_{m}},
$$

for any $z \in \mathbb{I}$. Since $m n_{m} \leq m, n_{m}=0$ or 1 . If $n_{m}=0$, then the left side is bounded by $C^{\prime}$; if $n_{m}=1$, then the left side is bounded by $C^{\prime}\left|g_{0}^{(m)}(z)\right|$. In both cases, the right hand side is $L^{2}$-integrable. This shows that $B^{(m)}$ is $L^{2}$ integrable. Similar conclusion holds when $X$ and $Z$ are dependent and the conditional density of $X$ given $Z$, namely, $\pi(x \mid z)$, have bounded $l$ th partial derivatives with respect to $z$, for $l \leq m$. Then (3.1) holds for $b=1$.

For any constant vector $v \neq 0, P\left(v^{T}(X-G(Z))=0\right)<1$, then we have $\Omega=E\left\{I(U)(X-G(Z))(X-G(Z))^{T}\right\}$ is positive definite, i.e., Assumption A3 holds. To see this, if $\Omega$ is not positive definite, then there exists a vector $v \neq 0$ such that $v^{T} \Omega v=0$. Since $I(U)$ has positive lower bound, we have $v^{T}(X-G(Z))=0$, a.s. Contradiction! Meanwhile, Proposition 2.5 implies that Assumption A4 and A5 will be satisfied given $h \asymp h^{*}$.

Since $g_{0}$ satisfies the condition in Corollary 4.5 , both $b_{z_{0}}$ in (3.4) and $c_{z_{0}}$ in the statement of Theorem 4.4 vanish. By $m>1+\sqrt{3} / 2$ and $h \asymp h^{*}$, it can be shown that the rate conditions in Theorems 3.1 and 4.4 hold. Therefore, all the assumptions in Theorems 3.1 and 4.4 are satisfied, and the corresponding conclusions hold.

S.16. A Computational Algorithm for the Constrained Smoothing Spline Estimate. Consider testing $H_{0}: x_{0} \theta+g\left(z_{0}\right)=0$ where $x_{0} \in(0,1)$ and $\theta_{0}$ are both one-dimensional. We demonstrate how to find constrained estimate of $\widehat{f}_{n, \lambda}^{0}=\left(\widehat{\theta}_{n, \lambda}^{0}, \widehat{g}_{n, \lambda}^{0}\right)$ under $H_{0}$, i.e., to find the solution to

$$
\arg \min _{(\theta, g): x_{0} \theta+g\left(z_{0}\right)=0} n^{-1} \sum_{i=1}^{n}\left(Y_{i}-X_{i} \theta-g\left(Z_{i}\right)\right)^{2} /\left(2 \widehat{\sigma}^{2}\right)+\lambda^{\prime} J(g, g) .
$$

Let $R(s, t)$ be the reproducing kernel function associated with the roughness penalty $J$. Under $H_{0}$, the quadratic function in (S.19) is rewritten as

$$
n^{-1} \sum_{i=1}^{n}\left(Y_{i}+X_{i} g\left(z_{0}\right) / x_{0}-g\left(Z_{i}\right)\right)^{2} /\left(2 \widehat{\sigma}^{2}\right)+\lambda^{\prime} J(g, g) .
$$

Let $\tilde{K}$ be the reproducing kernel for $H_{0}^{m}(\mathbb{I})$ associated with the usual inner product $\langle\cdot, \cdot\rangle_{0}$ defined in [35]. Define $\eta_{i}=\tilde{K}_{Z_{i}}-X_{i} \tilde{K}_{z_{0}} / x_{0}$, for $i=1, \ldots, n$. 
Then $\left\langle\eta_{i}, g\right\rangle_{0}=g\left(Z_{i}\right)-X_{i} g\left(z_{0}\right) / x_{0}$. It follows from Theorem 1.3.1 of [35] that the minimizer of (S.20) has the form $\widehat{g}_{n, \lambda}^{*}=\alpha+\sum_{i=1}^{n} \beta_{i} \mathcal{P} \eta_{i}$, where $\alpha, \beta_{i}$ are constants and $\mathcal{P}$ is the projection from $H_{0}^{m}(\mathbb{I})$ to the complement of the null space of $J$. It can be seen from Chapter 2 of [35] that $\mathcal{P} \tilde{K}_{z}=R_{z}$, for any $z \in \mathbb{I}$. Then $P \eta_{i}=R_{Z_{i}}-X_{i} R_{z_{0}} / x_{0}$. Let $Q$ be a symmetric $n \times n$ matrix with entries $Q_{i j}=R\left(Z_{i}, Z_{j}\right)-X_{j} R\left(Z_{i}, z_{0}\right) / x_{0}-X_{i} R\left(Z_{j}, z_{0}\right) / x_{0}+$ $X_{i} X_{j} R\left(z_{0}, z_{0}\right) / x_{0}^{2}, S$ be an $n$-vector with elements $S_{i}=1-X_{i} / x_{0}$. Let $Y=\left(Y_{1}, \ldots, Y_{n}\right)^{T}$ and $\beta=\left(\beta_{1}, \ldots, \beta_{n}\right)^{T}$. Minimizing (S.20) can thus be achieved by optimizing the following function of $\alpha, \beta$ :

$$
\|Y-S \alpha-Q \beta\|^{2}+\widehat{\sigma}^{2} n \lambda \beta^{T} Q \beta .
$$

The optimization of (S.21) can be achieved by similar arguments in [13]. Specifically, the solution has the form

$$
\beta=F_{2}\left(F_{2}^{T} Q F_{2}+n \lambda \widehat{\sigma}^{2} I\right)^{-1} F_{2}^{T} Y, \quad \alpha=\left(S^{T} S\right)^{-1} S^{T}\left(Y-\left(Q+n \lambda \widehat{\sigma}^{2} I\right) \beta\right) .
$$

\title{
REFERENCES
}

[S1] Johnson, W. P. (2002). The curious history of Faá di Bruno's formula. American Mathematical Monthly, 109, 217-234.

[S2] Pinelis, I. (1994). Optimum bounds for the distributions of martingales in Banach spaces. Annals of Probability, 22, 1679-1706.

[S3] Rudin, W. (1976). Principles of Mathematical Analysis. McGraw-Hill. New York.

[S4] Shang, Z. (2010). Convergence rate and Bahadur type representation of general smoothing spline M-Estimates. Electronic Journal of Statistics, 4, 1411-1442.

\author{
Department of Statistics \\ Purdue University \\ 250 N. University Street \\ WEST LAFAYETTE, IN 47907 \\ EMAIL: CHENGG@PURDUE.EDU; SHANG9@PURduE.EDU
}



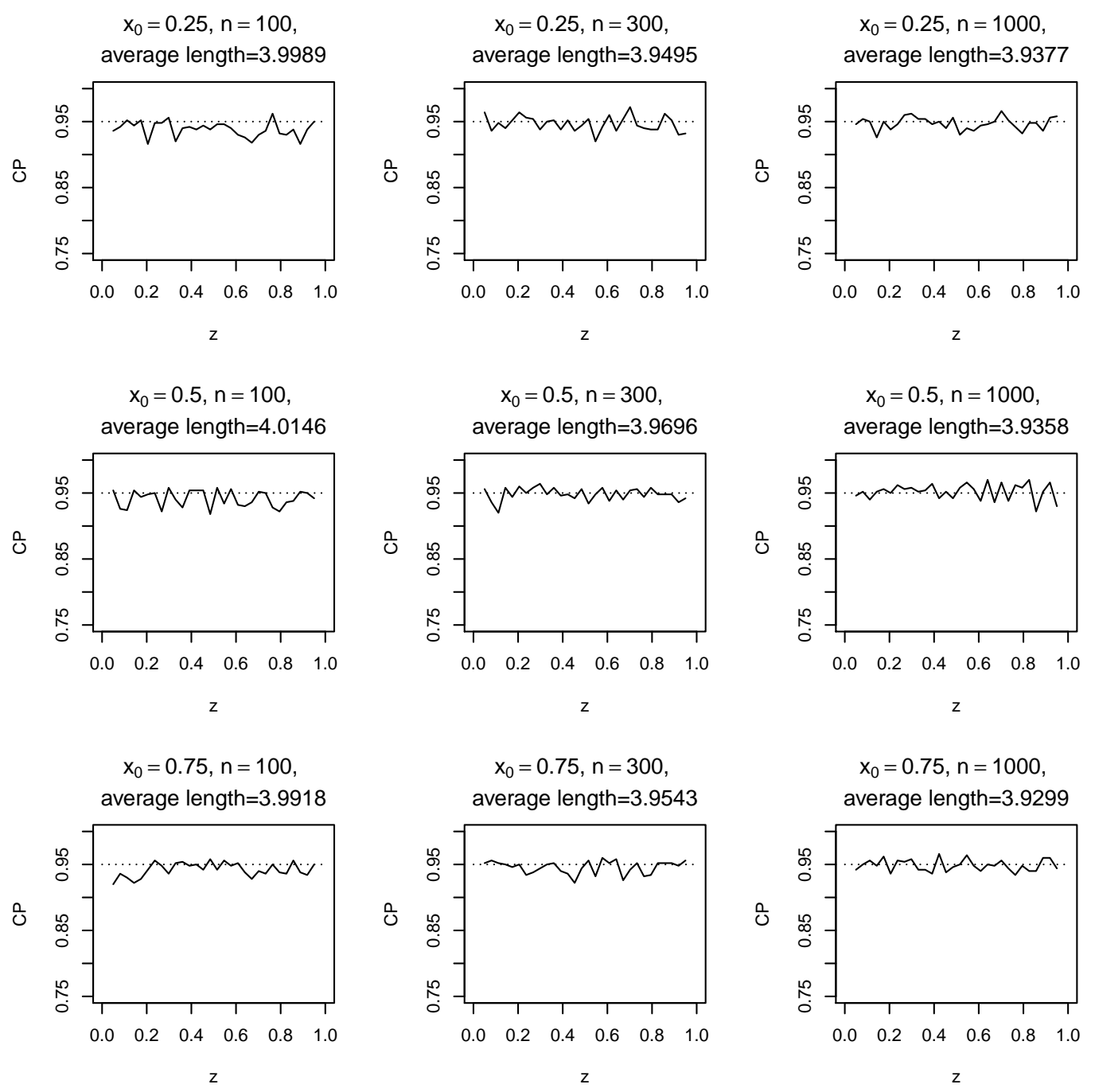

FIG 2. Coverage proportion of $95 \%$ prediction intervals in Case (I) of Example 5.1. 

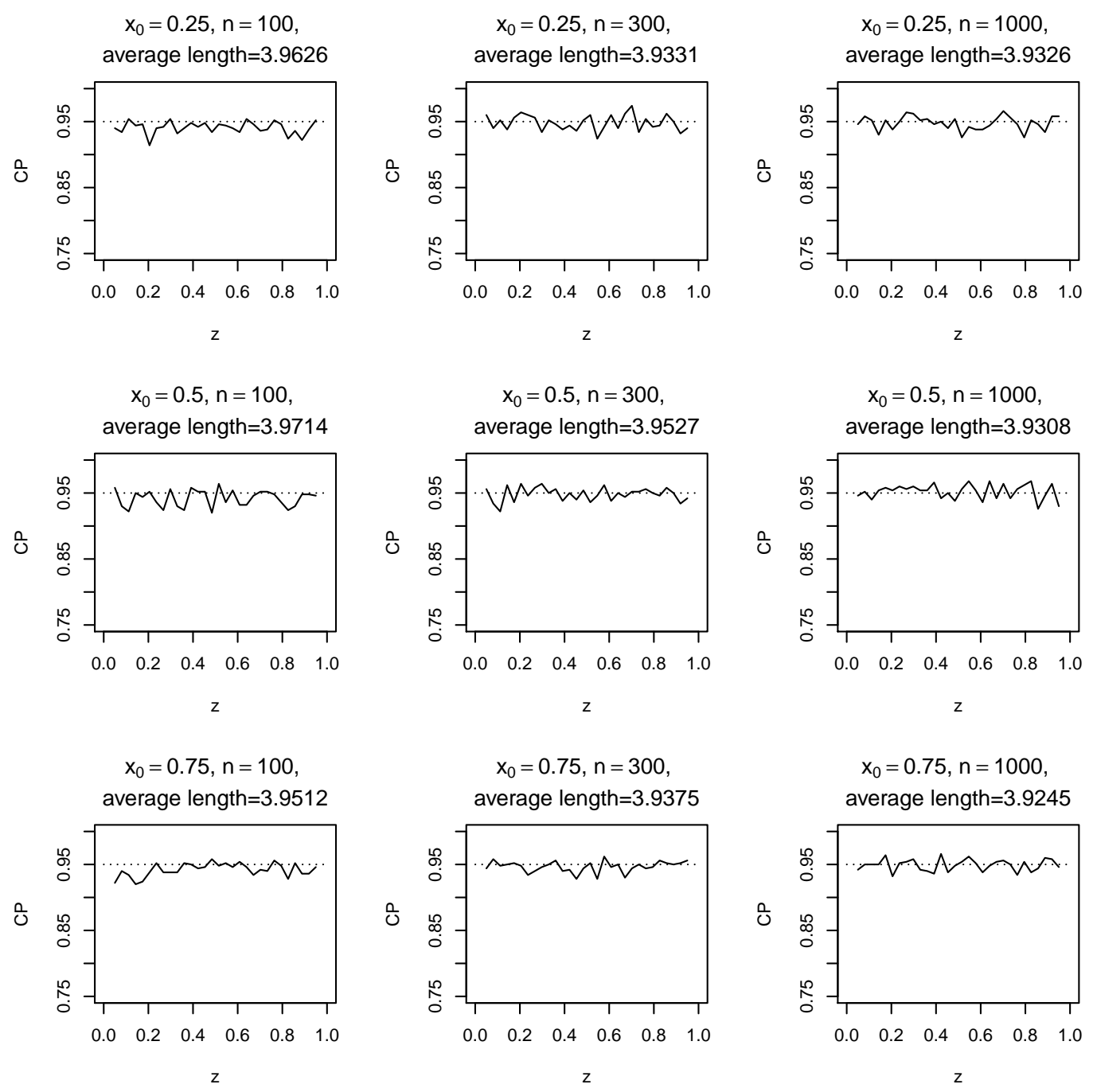

FIG 3. Coverage proportion of $95 \%$ prediction intervals in Case (II) of Example 5.1. 

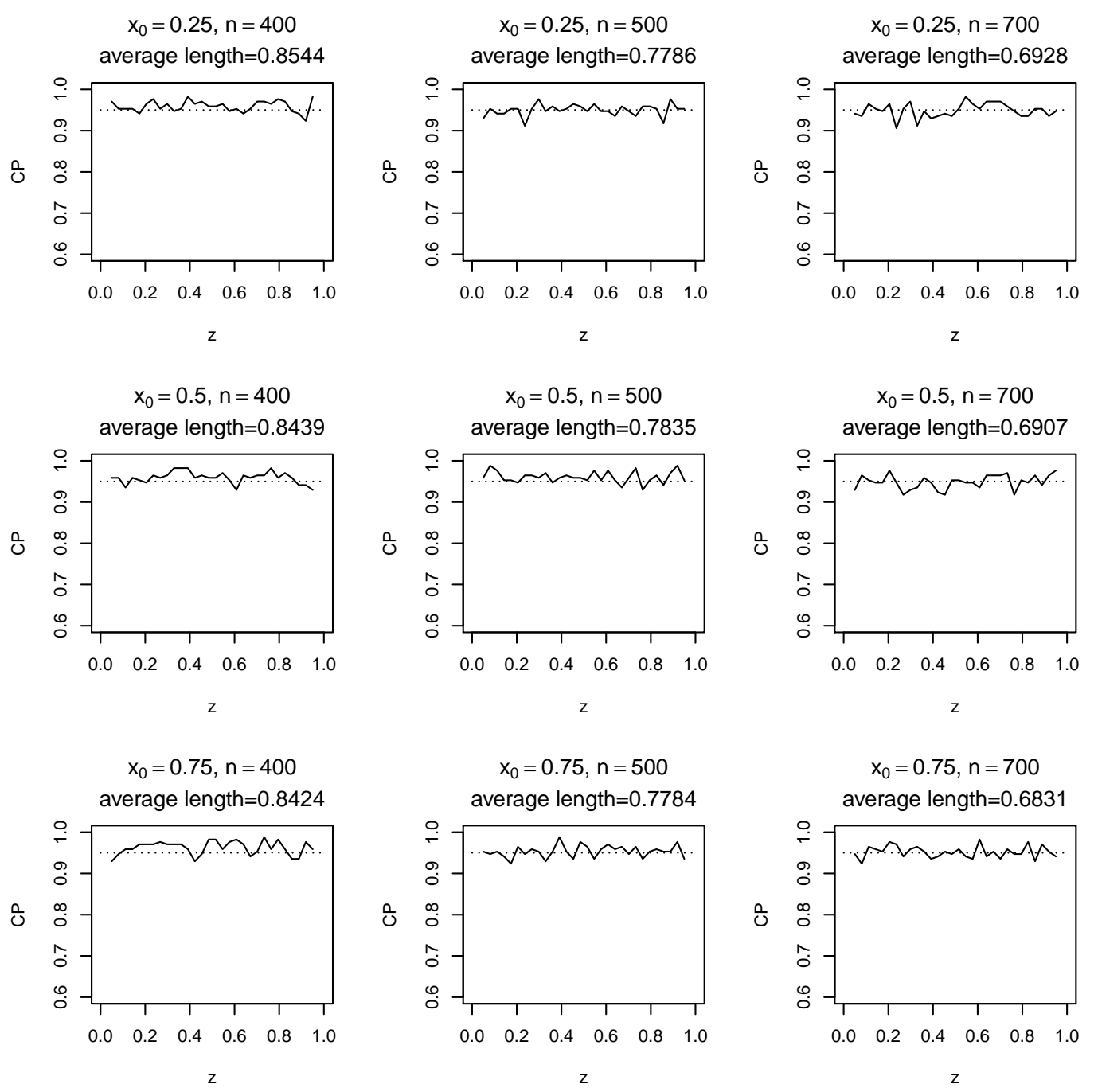

FIG 4. Coverage proportion of $95 \%$ CIs for the conditional mean constructed at a variety of $(x, z)$ values. 